\title{
Pore-network model of evaporation-induced salt precipitation in porous media: The effect of correlations and heterogeneity
}

DOI:

10.1016/j.advwatres.2017.12.004

\section{Document Version}

Accepted author manuscript

Link to publication record in Manchester Research Explorer

Citation for published version (APA):

Dashtian, H., Shokri, N., \& Sahimi, M. (2018). Pore-network model of evaporation-induced salt precipitation in porous media: The effect of correlations and heterogeneity. Advances in Water Resources, 112, 59-71.

https://doi.org/10.1016/j.advwatres.2017.12.004

Published in:

Advances in Water Resources

\section{Citing this paper}

Please note that where the full-text provided on Manchester Research Explorer is the Author Accepted Manuscript or Proof version this may differ from the final Published version. If citing, it is advised that you check and use the publisher's definitive version.

\section{General rights}

Copyright and moral rights for the publications made accessible in the Research Explorer are retained by the authors and/or other copyright owners and it is a condition of accessing publications that users recognise and abide by the legal requirements associated with these rights.

\section{Takedown policy}

If you believe that this document breaches copyright please refer to the University of Manchester's Takedown Procedures [http://man.ac.uk/04Y6Bo] or contact uml.scholarlycommunications@manchester.ac.uk providing relevant details, so we can investigate your claim.

\section{OPEN ACCESS}




\title{
Pore-network model of evaporation-induced salt precipitation in porous media: the effect of correlations and heterogeneity
}

\author{
Hassan Dashtian, ${ }^{a}$ Nima Shokri, ${ }^{b}$ Muhammad Sahimi ${ }^{a, *}$ \\ ${ }^{a}$ Mork Family Department of Chemical Engineering and Materials Science, University of South- \\ ern California, Los Angeles, California 90089-1211, USA \\ ${ }^{b}$ School of Chemical Engineering and Analytical Science, The University of Manchester, Manch- \\ ester M13 3PL, United Kingdom
}

\begin{abstract}
Salt transport and precipitation in porous media constitute a set of complex and fascinating phenomena that are of considerable interest to several important problems, ranging from storage of $\mathrm{CO}_{2}$ in geological formations, to soil fertility, and protection of pavements and roads, as well as historical monuments. The phenomena occur at the pore scale and are greatly influenced by the heterogeneity of the pore space morphology. We present a pore-network (PN) model to study the phenomena. Vapor diffusion, capillary effect at the brine-vapor interface, flow of brine, and transport of salt and its precipitation in the pores that plug the pores partially or completely are all accounted for. The drying process is modeled by the invasion percolation, while transport of salt in brine is accounted for by the convective-diffusion equation. We demonstrate that the drying patterns, the clustering and connectivity of the pore throats in which salt precipitation occurs, the saturation distribution, and the drying rate are all strongly dependent upon the pore-size distribution, the correlations among the pore sizes, and the anisotropy of the pore space caused by stratification that most natural porous media contain. In particular, if the strata are more or less parallel to the direction of injection of the gas that dries out the pore space (air, for example) and/or causes salt precipitation $\left(\mathrm{CO}_{2}\right.$, for example), the drying rate increases significantly. Moreover, salt tends to precipitate in clusters of neighboring pores that are parallel to the open surface of the porous medium.

*Corresponding author

E-mail addresses: Dashtian@usc.edu (H. Dashtian), nima.shokri@manchester.ac.uk (N. Shokri), moe@usc.edu (M. Sahimi)
\end{abstract}

Keywords: Evaporation, salt precipitation, pore-network simulation, correlation 


\section{Introduction}

Salt precipitation in porous media occurs in numerous systems, including in soil that represents a global issue affecting salinization of agricultural land (Nachshon et al., 2001, 2011; Schoups et al., 2005) and in geological formations that are being tested for $\mathrm{CO}_{2}$ sequestration (Baumann et al., 2014). Several mechanisms contribute to salt precipitation in porous media, the most important of which is drying and evaporation/wicking of saline water (Veran-Tissoires and Prat, 2014), as well as mineral replacement and reactions that contribute to salt crystallization (Dashtian et al., 2017) and growth in soil and rock (Noiriel et al., 2010). Salt precipitation damages porous media by reducing their porosity and permeability (Bacci et al., 2011), causing mechanical failure (Schiro et al., 2011). Thus, numerous theoretical and experimental investigations have been undertaken to understand the effect of various factors on drying of brine and salt distribution (Shokri et al., 2008; Shokri and Or, 2011; Norouzi Rad and Shokri, 2012; Norouzi Rad et al., 2013; Bergstad and Shokri, 2016) in order to develop methods for limiting the damage. In particular, Shahidzadeh-Bonn et al. (2010) investigated the effect of the kinetics of salt dissolution and crystallization on salt-induced damage to sandstones, while Flatt (2002) studied the effect of salt concentration on the extent of damage to porous media.

Salt precipitation is preceded by evaporation of saline water. Thus, many experimental studies of brine evaporation (Shahraeeni and Or, 2010; Shahraeeni et al., 2012; Shokri and Or, 2011) and precipitation of salt (Shokri et al., 2010; Bergstad and Shokri, 2016) at the pore scale have been reported, elucidating the effect on the drying rates of several factors, such as the pore-size distribution (PSD), connectivity of the pore space - the way pore bodies and pore throats are connected to one another - and the wettability. For example, experiments with various PSDs indicate that two types of efflorescence, crusty and patchy, may form on the surface of porous media (Veran-Tissoires and Prat, 2014) that affect the evaporation rates differently. Thus, the PSD is a major component of porous medium that should be considered in modeling of drying-induced salt precipitation.

But, despite the large number of theoretical and experimental studies of salt crystallization in porous media, there is still no comprehensive model and efficient numerical simulation method that can predict salt precipitation patterns in porous media at the pore scale, usually referred to as subflorescence. Salt crystallization in a porous medium is a complex molecular- 
scale phenomenon (Dashtian et al., 2017) that is influenced by the thermodynamic state of the system - temperature, pressure, and humidity - and other factors, such as the salt type and the morphology of the medium. Even repeating a given experiment in a sample porous medium undergoing salt crystallization does not guarantee the same precipitation patterns. Such complexities in the physics of the salt precipitation in porous media exacerbate the inherent difficulties of its modeling. The goal of our study is to propose a pore-network (PN) modeling approach that is capable of predicting the spatial distributions of brine, vapor and the precipitated salt.

To develop a physically-based PN model, one must take into account the mechanism by which the precipitated salt accumulates. Under the right conditions, $\mathrm{Na}^{+}$and $\mathrm{Cl}^{-}$ions in a solution give rise to nucleation and growth of salt crystals. Experimental studies by ShahidzadehBonn et al. (2010) demonstrated the effect of the salt type on the extent of damage to the pore space of porous media. They showed that the formation of the hydrated sodium sulfate crystals enhances the spreading power of the salt solution on the PN network of sandstone, giving rise to very rapid growth of the hydrated phase of sulfate and forming clusters. Sodium chloride, on the other hand, only forms anhydrous crystals, which explains why less efflorescence is observed compared to sodium sulfate. Shahidzadeh-Bonn et al. (2010) did not, however, consider the effect of the chemistry of the pore surface of porous media, or the co-existence of several types of salt. In our PN model, we consider only the formation of subflorescence and, thus, we work with $\mathrm{NaCl}$ as the salt in the study.

Some experimental and theoretical studies indicate that salt accumulates in a very narrow layer at the soil-atmosphere interface, where the initial evaporation occurs, after which precipitation within the pore space is limited. On the other hand, experimental studies of Roels et al. (2014) with core samples indicated that capillary-driven flow does not have substantial effect on salt precipitation, because of which accumulation of salt near the core surface was low. Their computed-tomography images after drying indicated uniform salt distribution inside the core. Börnhorst et al. (2016) reported that high evaporation rates limit transport of solids on the external surface, leading to a more uniform precipitation distribution, whereas low drying rates result in strong solid accumulation on the porous medium's external surface, but that small throats reduce the solute transport and restrict solid accumulation on the surface. One must also recognize the self-enhancing of salt growth and salt transport in water films (Miri 
et al., 2015). Aggregated salt crystals form a microporous medium (Malmir et al., 2016a,b, 2017) with a high degree of capillarity in each throat that enhances solute transport through imbibing brine over long distances. But, since such phenomena as the growth of salt crystals at the drying interface occur at the molecular scale (Dashtian et al., 2017), it is not possible to include them in a PN model. Instead, one must resort to molecular dynamics simulation (Dashtian et al., 2017).

Kim et al. (2013) reported on two distinct salt crystallization in synthetic porous media. One was large bulky crystals that form away from the vapor-liquid interface, while aggregated, randomly-oriented crystals that form near the drying interface constituted the second type. In addition, experiments in circular capillary tubes have shown that salt precipitation is also affected by the boundary conditions imposed on the system. When the two ends of capillary tubes are open, salt crystals tend to migrate even outside the tube and form a patchy packing. In closed-end capillaries, however, salt crystallization occurs inside the tubes (Miri et al., 2015). Therefore, the question of whether salt precipitates mainly on the external surface of a porous medium, or more uniformly in its pore space, depends on the medium's morphology, the competition between convection and capillarity, and the boundary conditions. In the PN model that we describe below, salt precipitate occurs inside the pore throats and, after precipitation, its crystals do not migrate from one throat to other throats.

At the field scale salt precipitation is also influenced by the initial spatial distributions of the porosity and permeability of a porous formation (Zeidouni et al., 2008; Celia et al., 2011). Several experimental studies of salt precipitation in geological formations during $\mathrm{CO}_{2}$ injection have also been reported (Muller et al., 2009; Wang et al., 2009; Bacci et al., 2011; Ott et al., 2011; Peysson et al., 2014), indicating that salt precipitation reduces permeability and porosity in Berea sandstone. This supports the view that in such formations crystallization occurs inside the pore throats in the pore space, rather than migration to the outside surface. Given such insights, the effect of such distributions on the efflorescence formation has also been studied (Veran-Tissoires and Prat, 2012; Veran-Tissoires et al., 2012). In particular, Sghaier et al. (2014) reported on the influence of evaporation flux from the boundary on the subflorescence formation and growth, while the effect of grain angularity was studied by Norouzi Rad and Shokri (2014). The porous media used by Sghaier et al. (2014) and Norouzi Rad and Shokri (2014) represented, however, isotropic medium, and did not also include the correlations in 
the grain or pore sizes that are prevalent in natural porous media (Sahimi, 2011). One major purpose of our study is to show the effect of pore-size correlations on drying and the precipitation patterns.

Models of evaporation and salt transport and precipitation in porous formations can be divided into two groups. In one group are the thermodynamic models of salt deposition in Earth's crust; see, for example, Toner et al. (2015). The second groups of models contains two subgroups. Continuum models of fluid flow and transport (Huinink et al., 2002; Guglielmini et al., 2008) - those that are based on the classical averaged equations of hydrodynamics and transport - belong to the first subgroup. They are capable of modeling evaporation and salt precipitation at the field scale. They include models for the evolution of salt crystals (Le et al., 2009) and salt concentration in liquids (Mahadevan et al., 2006) in porous media. They only predict, however, the location of the maximum salt concentration inside porous medium. Salt precipitation and invasion of the pore space by a gas (e.g. air) are not included in such models.

Though continuum models provide valuable insights, they cannot take into account the effect of the pore-scale heterogeneity of a pore space, which is of utmost importance because, as already pointed out, drying and salt precipitation are pore-scale phenomena that are influenced strongly by pore-scale heterogeneity. Thus, one needs to develop PN models in order to study the effect of pore-scale disorder on evaporation and salt precipitation. Once the phenomena are understood at the PN scale, one can up-scale the models to much larger porous media. The PN models have been used extensively for decades to study various phenomena in porous media (for reviews see Sahimi, 2011; Blunt, 2017). Recent applications of the PNs include coating of surface of paper (Ghassemzadeh et al., 2001; Ghassemzadeh and Sahimi, 2004), transport and separation of gaseous mixture in nanoporous membranes (Chen et al., 2008; Rajabbeigi et al., 2009; Mourhatch et al., 2010a), flow permporometry for measuring the PSD of a mesoporous medium (Mourhatch et al., 2010b), and simulation of multiphase fluid flow in reservoir rock (Blunt et al., 2002, 2013; Piri and Blunt, 2005a,b; Blunt, 2017).

Development of the PN model of evaporation-induced salt precipitation involves simulation of the drying process. Slow drying of porous media is an invasion percolation (IP) process (for a simple introduction to the IP see Ebrahimi, 2010), whereby the drying front invades a porous medium (Shaw, 1987) according to the least capillary pressure that a nonwetting fluid (air) requires to enter a pore throat filled by saline water (see below). Using the IP process, many 
PNs have been developed for simulating drying (Daian and Saliba, 1991; Prat, 1995; Laurindo and Prat, 1998; Yiotis et al., 2001, 2006; Metzger et al., 2007; Surasani et al., 2007). The performance of PN models of evaporation in porous media have been tested in the previous studies. In our modeling, we use the same approach to simulate the evaporation part of the process.

Thus, the goal of the present study is developing a PN model for evaporation, brine transport and salt precipitation in porous media, and presenting the results of extensive PN simulations by which we have computed the various important properties of the phenomena. To our knowledge only Bönhorst et al. (2016) presented a PN model of precipitation of solids in porous media, which is presumably applicable to salt precipitation. Their model differs, however, from what we present in this paper. In their work transport of the solids is by diffusion, rather than by the convection-diffusion process. It is known, however (Idamkm and Sahimi, 1987, 1991; Sahimi and Imdakm, 1991), that if the particles are relatively large, diffusion is not an effective mode of transport, and the range of the particle sizes that their model is applicable for is not clear to us. At the same time, even if diffusion is an effective mechanism of the particles, it could be so only for porous media whose pore throats are very small, so that convection may be ignored, or that the flow field is very slow. Moreover, the important effect of the heterogeneity of porous media, which is due to the PSD and the correlations between the pore sizes is taken into account by our PN model, whereas this important effect was mostly ignored in the model by Bönhorst et al. (2016). Indeed, a major aspect of salt precipitation at the pore scale is the effect of the heterogeneity.

The rest of this paper is organized as follows. In the next section we describe the PN model. The details of the models of drying and salt transport and precipitation are presented in Sec. 3, while the computational algorithm is described in Sec. 4, followed by the presentation and discussion of the results in Sec. 5. The paper is summarized in Sec. 6.

\section{Pore-network model}

We use a square network with a pore connectivity of 4 . Extension of the model to 3D is straightforward but, relative to 2D networks, computationally expensive and will be reported elsewhere. Pressure and concentration gradients in pore bodies are ignored, the standard practice in many PN modelings. Thus, each pore body is characterized by a single value of 
liquid (or vapor) concentration and pressure, the calculation of which is described below. As a result, when we refer to the PSD of the PN, we mean the size distribution of the pore throats. The pore throats are assumed to be cylindrical. So long as the effect of thin liquid films on the internal surface of the pore throats is insignificant, the assumption of cylindrical throat is accurate, and has been used many times in the past. In the present paper in which we are interested in precipitation of salt, the thin films are indeed unimportant.

An important aspect of the morphology of porous media that has not been considered in the simulation of drying is the correlations between the size of the pore throats. The existence of such correlations in the porosity distribution of field-scale porous media was first demonstrated by Hewett (1986), followed by similar work by Neuman (1994) for the spatial distribution of the permeability of the same type of porous media. In fact, such correlations manifest themselves over multitudes of scales (Sahimi and Mukhopadhyay, 1996; Mukhopadhyay and Sahimi, 2000; Dashtian et al., 2011, 2015). Using experiments and PN simulations, Knackstedt et al. (2001) demonstrated that, even at the core scale, the computed properties of multiphase flow in porous media agree quantitatively with the experiments when such correlations are taken into account. To include the effect of the correlations in the size of the throats, we used a fractional Brownian motion (FBM) that has been shown to not only represent well the distribution of the permeabilities in field-scale porous media (for a review see Sahimi, 2011), but also the correlated distribution of the throat sizes in cores (Knackstedt et al., 1998). The two-point correlation function $C(r)$ of a FBM is given by, $C(r)=C_{1} r^{2 H}$, where $C_{1}=C(r=1)$, and $H$ is the Hurst exponent. The correlations are positive or persistent if $H>1 / 2$, and negative or anti-persistent if $H<1 / 2$. In the limit $H=1 / 2$ the successive increments of a FBM are completely uncorrelated. An efficient method for generating a FBM array is through its power spectrum $S(\boldsymbol{\omega})$, which for $2 \mathrm{D}$ systems is given by

$$
S(\boldsymbol{\omega})=\frac{a}{\left(\omega_{x}^{2}+\omega_{y}^{2}\right)^{H+1}},
$$

where $a$ is a constant, while $\omega_{x}$ and $\omega_{y}$ are the Fourier components in the $x$ and $y$ directions. To introduce anisotropy (layering) in the distribution, we follow Ansari-Rad et al. (2012) and generalize the power spectrum to

$$
S(\omega)=\frac{b}{\left(\beta_{x} \omega_{x}^{2}+\beta_{y} \omega_{y}^{2}\right)^{H+1}} .
$$


Here, $\beta_{x}$ and $\beta_{y}$ are the anisotropy parameters, and $b$ is a constant. To generate anisotropy induced by layering with the layers being parallel to the $x$ direction, we set $\beta_{x} / \beta_{y}>1$, and similarly for the layers being parallel to the $y$ direction. Figure 1 presents examples of the heterogeneities generated by the model.

\section{Pore-network model and the governing equations}

As the evaporation proceeds, the vapor-brine interface advances in the pore space, and salt begins to precipitate, which we assume to be mainly $\mathrm{NaCl}$. Thus, one must simulate both the drying and salt transport and precipitation. The computations consist of two distinct parts. One is simulation of the drying process by the IP algorithm. The PN's top row is assumed to be a layer whose throats are initially empty and through which a gas (such as air or $\mathrm{CO}_{2}$ ) flows into the pore space, while the other sides are sealed. Brine flows in the pore space's throats, while the vapor diffuses to the boundary layer and induces further evaporation. In the IP model a gas invades the brine-saturated porous medium throat-by-throat according to the lowest capillary pressure needed for entering a throat at the interface between the vapor and liquid. The capillary pressure for entering a throat of radius $r_{i j}$ between pores $i$ and $j$ at the liquid-vapor interface is given by

$$
P_{c, i j}=\frac{2 \sigma}{r_{i j}},
$$

where $\sigma$ is the surface tension. Each time one or a few throats are dried out by the IP algorithm, we switch to the second part of the simulation, namely, transport of brine and precipitation of salt, described below.

At every step of the simulation we identify the state of the pores, which is determined by the filling state of the pore throats connected to them: if at least one neighboring throat does not contain brine, it is a vapor pore; otherwise, it is a liquid pore. A throat can be filled, fully or partially, with brine, or it can contain vapor that is leaving it through a path of pores and throats that are also filled by the vapor. A vapor pore can be either at equilibrium or at an unknown vapor pressure (to be determined). In reality, equilibrium vapor pressure prevails only at the menisci between brine and the vapor, as shown in Fig. 2, but we assume that a pore is at equilibrium vapor pressure if at least one of its neighboring throats still contains brine, even if it is only partially so. Therefore, diffusion of vapor between menisci of partiallyfilled throats and the adjoining vapor pore happens without any resistance. Our preliminary 
numerical simulation indicated that the assumption leads to slightly, only about 5 percent, overestimates of the drying rate, but simplifies the computations greatly.

Once the states of pores and throats are identified, the second stage of the simulation, namely, modeling of transport of brine throughout the PN and the resulting salt precipitation, begins. After one or a few steps of the IP that advance the drying front, we solve the governing transport equations. Assuming that the flow is slow enough, the liquid flow rate $q_{i j}^{(l)}$ in a throat $i j$ that connects pores $i$ and $j$ is given by the Hagen-Poiseuille equation

$$
q_{i j}^{(l)}=\frac{\pi r_{i j}^{4}}{8 \nu^{(l)} \ell_{i j}}\left[P_{i}^{(l)}-P_{j}^{(l)}\right],
$$

where the liquid viscosity $\nu^{(l)}$ is assumed to be constant, as we simulate an isothermal process. Here, $P_{i}^{(l)}$ is the liquid pressure in pore $i$, and $\ell_{i j}$ is the length of the throat $i j$. Yiotis et al. (2001) showed that both advection and viscous effects affect the drying patterns and rates. Since there is no liquid accumulation in the pores, we must have

$$
\sum_{\{i j\}} \rho^{(l)} q_{i j}^{(l)}=0
$$

with $\rho^{(l)}$ being the brine's mass density. The sum is over the set of the throats $\{i j\}$ that are connected to pore $i$.

The vapor is transported by diffusion and, thus, we use the equation for evaporation in the so-called Stefan tube (Bird et al., 2007) to calculate its mass flow rate in the throats. Ignoring the pressure in the vapor-filled throats, vapor mass conservation at the pores must hold, implying that,

$$
\sum_{\{i j\}} \rho^{(v)} q_{i j}^{(v)}=\sum_{\{i j\}} S_{i j} \frac{D^{(v)}}{\ell_{i j}} \frac{P M^{(v)}}{R T} \ln \left[\frac{P-P_{i}^{(v)}}{P-P_{j}^{(v)}}\right]=0,
$$

where $D^{(v)}$ is the vapor diffusion coefficient, $M^{(v)}$ its molecular weight, $P$ is the total pressure in the vapor phase, and $\rho^{(v)}$ is the vapor mass density. We used, $\rho^{(v)}=8.12 \times 10^{-4} \mathrm{gr} / \mathrm{cm}^{3}$ and $D^{(v)} \approx 2.6 \times 10^{-5} \mathrm{~m}^{2} \mathrm{~s}^{-1}$, both corresponding to $298 \mathrm{~K}$. The vapor concentration at the vaporliquid interface is equal to the equilibrium concentration, which is zero outside the boundary layer of the PN. Writing down Eqs. (4) - (6) for all the pores in the liquid and vapor phases results in two sets of equations that govern the pressures in the pores in the two phases, which are solved by the conjugate-gradient method. The pressure at the liquid-vapor interface is 
assumed equal to the equilibrium vapor pressure, $P^{*}=2339 \mathrm{~Pa}$. At a meniscus between the brine and vapor the Kelvin equation describes the dependence of parameters on the vapor pressure:

$$
\ln \left[\frac{P^{(v)}}{P^{*}}\right]=-\frac{2 M^{(v)} \sigma}{R T r_{i j} \rho^{(l)}}=-\frac{P_{c} M^{(v)}}{R T \rho^{(l)}},
$$

where $R$ is the gas constant, and Eq. (3) was used. Note that the dissolved salt increases the osmotic potential $\psi$, which in turn decreases the saturation vapor pressure of the saline water (Battistelli et al., 1997; Jambhekar et al., 2015). In addition, capillary pressure might also lower the vapor pressure. $\psi$ can be easily computed via, $\psi=R T \rho^{(l)} \ln \left(x^{(s)}\right)$, where $x^{(s)}$ is the solubility-limit mole fraction of salt in water. One can include the effect of osmotic pressure by replacing $P_{c}$ in Eq. $(7)$ by $\left(P_{c}+\psi\right)$ (Jambhekat et al., 2015), but the effect is believed to be small and, thus, we ignore it.

Transport of salt through a porous medium is by convection and diffusion (Shokri, 2014). The electroneutrality of brine implies that the concentration $C$ of the salt at time $t$ is governed by convection-diffusion equation (CDE):

$$
\frac{\partial C}{\partial t}=D_{e} \frac{\partial^{2} C}{\partial x^{2}}-v_{i j} \frac{\partial C}{\partial z}
$$

where $v_{i j}$ is the mean liquid velocity in the throat $i j$, and $D_{e}$ is the effective diffusion coefficient in the solution. Numerous studies have proposed various methods for including the effect of diffusion in flow through PNs (Sahimi and Imdakm, 1988; Koplik et al., 1988; Sorbie and Clifford, 1991; Bejeljic et al., 2004). One can simply take $D_{e}$ to be the molecular diffusivity $D_{m}$, which means that the throats must be narrow enough that the concentration at any given axial position $z$ within the throats is single-valued. Doing so simplifies the computations.

Alternatively, one may take $D_{e}=D_{L}$, where $D_{L}$ is the Taylor-Aris dispersion coefficient (Taylor, 1953; Aris, 1956). The choice $D_{e}=D_{L}$, which we make in this paper, entails imposing some constraints. According to Mehmani and Balhoff (2015) $D_{L}$ can be used in a PN simulation if the aspect ratio $A=\ell_{i j} / r_{i j}$ of the throats is 10 or larger. Note also that it has often been assumed and there is experimental evidence (Sahimi, 2011) that, $\ell_{i j} \propto 1 / r_{i j}$. Given that the throats' radii are typically small, $\ell_{i j}$ are typically large, which is what we also use. Thus, the condition for using the Taylor-Aris dispersion is satisfied, at least approximately. In a throat ij $D_{L}$ is given by

$$
D_{L}=D_{m}+\frac{r_{i j}^{2} v_{i j}^{2}}{48 D_{m}}
$$


One can show that the salt concentration near the liquid-vapor interface is maximum (Huinink et al., 2002; Guglielmini et al., 2008). Thus, salt precipitates mostly at the interface throats. In the liquid-filled pores $i$ the net accumulation of salt at time step $n$ is given by

$$
\sum_{\{i j\}} S_{i j} J_{i j}=\sum_{\{i j\}} v_{i j} \frac{C_{i}^{n+1}-C_{i}^{n}}{\Delta t},
$$

where $J_{i j}=v_{i j} C_{i}-D_{L} \partial C_{i} / \partial z=v_{i j} C_{i}-D_{L}\left(C_{j}-C_{i}\right) / \ell_{i j}$ is the mass flux in the throat $i j$ over a time step $\Delta t$. If a throat contains partially the vapor that can escape (i.e., it is connected to other pores and throats that are filled with vapor), then, mass balance will take the mass flow rate of the vapor in that throat into account. Writing the brine mass balance for each pore $i$ yields a set of equations for the concentrations $C_{i}$ of the salt that we also solve by the conjugate-gradient method.

\section{Computational algorithm}

Given the above formulations, the computational algorithm is as follows.

(i) The pressure distribution in the vapor phase is computed.

(ii) The mass evaporation rate in each throat and that from the boundary layer are calculated.

(iii) The pressure distribution in the liquid phase and, therefore, the mean flow velocity and the local dispersion coefficient $D_{L}$ in each throat are computed.

(iv) The time step for advancing the vapor-liquid interface by the IP algorithm is selected, following Hekmatzadeh et al. [2016]. There are two time scales in the problem. One is associated with evaporation, while the second one is the time scale over which one solves the CDE. We use the smaller of the two. Thus, the evaporated mass from all the throats in the drying front containing liquid throats, which leaves the $\mathrm{PN}$, is computed. The throat at the interface with the lowest capillary pressure is one with the largest radius. The time step for emptying that throat is given by

$$
\Delta t=\frac{\rho^{(l)} V_{i j}}{\sum_{\{i j\} \in \mathcal{L}_{D}} M_{i j}},
$$

where $V_{i j}$ is the volume of the throat $i j, M_{i j}$ is the mass rate of evaporation, and the sum is over all the throats $\{i j\}$ that are at the drying front $\mathcal{L}_{D}$.

(v) The CDE is solved in the brine part of the PN in order to compute the salt concentration in the throats. 
(vi) As drying continues, salt concentration in the liquid phase increases. As already pointed out, the solution of the CDE for salt transport in porous media indicates that salt concentration at the drying front is maximum (Huinink et al., 2002; Guglielmini et al., 2008). Thus, salt concentrations in the throats next to the drying front $\mathcal{L}_{D}$ are compared with a threshold concentration for its precipitation. If they exceed the threshold, which we take it to be the solubility limit of salt in water at room temperature (36 percent in mass), then, salt precipitates in such throats. Note also that preliminary simulations indicated that the salt content of practically no throat away from the interface exceeds the saturation limit. Then, the amount of precipitated salt in the throats is calculated as the difference between the current salt concentration in the throats and the saturation concentration. The precipitated salt forms a porous packing of its crystals and, therefore, it would take some time to plug a throat completely. Thus, the volume of the precipitated salt in each throat is calculated and subtracted from the throat's volume, and its radius is updated, assuming that salt precipitates uniformly. Depending on its size and the amount of precipitated salt, the throat can either become partially or completely plugged.

(vii) The IP algorithm advances further the drying front in the PN by one or a few throats, and the procedure is repeated until the evaporation rate from the boundary layer is negligible.

We note that recent work (Desarnaud et al., 2014) indicated that the precipitation threshold may be as high as 1.5 times the solubility limit. But, this would not make any difference to the PN model, as the threshold may be set at any value.

The PN is initially saturated with brine at $30 \% \mathrm{w}$. $\mathrm{NaCl}$. All the results presented below represent averages over at least 10 realizations of the PN. As discussed in Section 2, a FBM algorithm was used to generate 2D PNs with two values of the Hurst exponents $H$ and three values of $\beta_{x} / \beta_{y}$. Table 1 presents the numerical values of $H, \beta_{x} / \beta_{y}$, and the mean throat size (radius) for each case.

\section{Results and discussion}

Let us first inspect the effect of the PSD distribution and the correlations on the spatial distribution of the pore throats that are either filled with brine, or with the vapor, or are partially or completely plugged by the precipitated salt. Shown in Figures 3 is the evolution of 
Table 1: Pore size distribution properties of different cases.

\begin{tabular}{cccc}
\hline$H$ & $\beta_{x} / \beta_{y}$ & Initial mean throat size $(\mu m)$ & Final throat radius $(\mu m)$ \\
\hline 0.3 & 1 & 70.5 & 53.2 \\
0.3 & 4 & 74.1 & 55.9 \\
0.3 & $1 / 4$ & 67.7 & 51.1 \\
0.7 & 1 & 104.5 & 74.4 \\
0.7 & 4 & 110.1 & 88.4 \\
0.7 & $1 / 4$ & 101.1 & 66.7 \\
\hline
\end{tabular}

the phase distribution in an isotropic $\mathrm{PN}\left(\beta_{x}=\beta_{y}\right)$ with the Hurst exponent $H=0.7$, implying positive correlations between the sizes of the throats. In particular, Fig. 3(a) corresponds to the stage in which the drying rate is essentially constant (this will be discussed quantitatively shortly). Close to the open end, the throats empty (dry out) at a faster rate than those deeper in the pore space. Since the correlations in the PSD are positive, the throats with similar sizes are clustered together, and the drying patterns mimic the underlying connectivity of the throats. This is manifested by the large clusters that are clearly visible in Figure 3. Moreover, due to the isotropy of the pore space, the drying front advances in both directions, shown in Figures $3(\mathrm{~b})$ and 3(c).

Next, consider anisotropic (stratified) porous media. Figures 4 present the evolution of the spatial phase distribution with $\beta_{x} / \beta_{y}=1 / 4$, so that the throats with similar sizes tend to extend orthogonal to the open end of PN, as also indicated by Figure 1(b). Thus, preferential evaporation in the throats with similar sizes that are in the $y$ direction enhances the advancement of the drying front deep into large throats, while the liquid phase stays connected to the surface. AS a result, the drying front stretches out to the bottom of the PN faster than the isotropic porous media shown in Fig. 3; see Figs. 4(b) and 4(c). In the case of $\beta_{x} / \beta_{y}=4$, the strata are parallel to the open end of the PN. Thus, the throats with similar sizes cluster together and extend parallel to the open end. As depicted in Figs. 5(b) and 5(c), the drying patterns mimic the direction of the strata. Altogether, Figs. 3-5 demonstrate a universal effect in that they show the strong effect of positive and extended correlations on the drying patterns, and the fact the clusters of dried out throats form based on the underlying correlations.

Salt precipitation does not, however, follow a universal pattern in all the three cases. But, 
looking at the throats that are partially or completely plugged in Figs. 3 - 5, it can be seen that salt tends to precipitate in neighboring throats that are parallel to the open end of the PN.

As reviewed comprehensively by Sahimi (2011), extensive data indicate that if the correlations in porous media are of the FBM type, then the corresponding Hurst exponent $H$ is often less than 0.5, implying negative correlations between the throats' sizes and, therefore, a much more heterogeneous pore space than when $H>0.5$. Therefore, we also studied the cases in which $H=0.3$, with the corresponding means of the PSD presented in Table 1 . When the correlations are negative, a larger throat size is more likely to have small throats as its neighbors, and vise versa. The resulting evolution of the phase distributions are shown in Figs. 6 - 8 that correspond, respectively, to $\beta_{x} / \beta_{y}=1,1 / 4$ and 4 . Comparison of these figures with their counterparts when $H=0.7$ demonstrates the effect of correlations and PSD. Consider, for example, Figs. 6, and compare them with those in Fig. 3. Whereas clustering of the throats type - liquid filled, vapor filled, or partially or completely plugged - is clearly visible in Figs. 3 (and likewise in Figs. 4 and 5), the negative correlations essentially destroy such clustering effects.

Figure 9 presents the phase distribution in a $\mathrm{PN}$ in which there is no correlation between the size of the throats, but with the same range of throat sizes as before. The differences between the evolution of the phase distribution in Fig. 9 with those shown in Figs. 3-8 for $H=0.7$ are evident. In particular, the number of clustered brine-filled throats, as well as isolated liquid filled throats, is much higher in the case of random PN than when the morphology of the pore space is correlated.

We now make a quantitative comparison between the various cases whose phase distribution evolution has been described and discussed so far. An important property is the brine saturation $S$ and its evolution with the time. Figures 10 and 11 show the transversely-averaged (averaged over the $x$ direction in Figs. 3-9) saturations in the same six PNs of Figs. 3-9, computed at four times with the colors showing the range of variations over multiple realizations and the black curves indicating the averages over all the realizations. As one may expect based on Figs. 3-8, the correlations affect the saturation distribution strongly.

At any given time there exist three distinct regions in Fig. 10: one is associated with zero saturation over a range of the distance from the open end of the porous medium. The 
second region represents a transition zone in which $0<S<1$, while in the third region the medium is fully saturated, $S=1$. Note the stronger effect of positive correlations with $H=0.7$ on the saturation profiles, indicated by the fact that for negative correlations $(H=0.3)$ the range of variations in the saturations over multiple realizations is narrower. The effect is most pronounced when the porous medium is isotropic, as the advancing drying interface does not have any preferred direction to move. Another notable difference is the depth at which the transition zone ends, i.e. where $S=1$. For example, consider the time at which the liquid saturation is $40 \%$ (dark blue color). The depth at which the transition zone ends is 40 (in units of the throats' length) for $H=0.7$ and $\beta_{x} / \beta_{y}=1 / 4$, whereas it is 30 for $\beta_{x} / \beta_{y}=4$. Similar patterns exist with negative correlations, as shown in Fig. 10. The ranking of the depth at which the transition zone ends is $\beta_{x} / \beta_{y}=4<\beta_{x} / \beta_{y}=1<\beta_{x} / \beta_{y}=1 / 4$.

Under isothermal condition the evaporation rate depends on such driving forces as the capillary and viscous forces (Shokri and Or, 2011; Lehmann et al., 2008). The capillary pressure depends, of course, on the PSD. Scherer (1990) discussed the difference between the capillary pressures of large and small throats that result in flow of the liquid to the surface of a porous medium, keeping the evaporation close to the early stages, which is called capillary pumping. The evaporation rate at the open side of the porous medium connected to the boundary pores is given by

$$
\dot{m}^{(v)}=\sum_{i \in \text { boundary }} \sum_{j \in\{i\}} S_{i j} \frac{D^{(v)}}{L} \frac{P M}{R T} \ln \left[\frac{P-P_{i}^{(v)}}{P-P_{j}^{(v)}}\right] .
$$

Figure 12 compares the evolution of the evaporation rates, usually called the drying curve, which were computed for the six types of the PNs of Figs. 3-8, averaged over multiple realizations of the PNs and rescaled by the number of pores in the open boundary of the PNs and the initial drying rate. The qualitative trends of Figure 12 are in complete agreement with the recent experimental data of Shokri-Kuehni et al. (2017).

The results in Figure 12 manifest the three main stages of drying and precipitation: (i) the initial constant evaporation; (ii) the relatively fast intermediate falling rate, and (iii) the residual slow rate of evaporation. The first stage is essentially independent of the morphology of porous media and is controlled by the external conditions. It is visible in Fig. 12 as a very short period in the initial time steps. Evaporation in the intermediate stage is dictated by the ability of the porous medium to supply the evaporation zone with brine, which in turn depends 
strongly on the morphology of the pore space, and in particular the PSD, the correlations, and the pore connectivity, and lasts much longer than the initial stage. Comparing all the cases, the intermediate stage with $\beta_{x} / \beta_{y}=4$ and $H=0.7$ has the highest evaporation rate, as shown in Fig. 12. As we discussed earlier when we described Figure 4, in this case the liquid stays connected to the surface of the pore space and, therefore, evaporation can happen in throats near the surface. Evaporation from the open end of the pore space dominates drying from the throats deeper into the pore space.

To better understand the results, consider flow of brine through the PN between two points separated by several throats. If the throats' sizes between the two points are positively correlated, there will be smaller resistant against the flow. In the PN in which the strata are parallel to the $y$ direction - Figs. 12(b) and 12(e) - the falling rate is slower and, thus, this stage lasts until almost the end of the process, followed by fast declining. The third stage emerges when the surface zone has completely dried out, so that further flow of liquid through it effectively ceases.

There are some studies (e.g., Or et al., 2013) that define the drying in two stages. Depending on the porous media and the evaporation condition, there might be other phenomena, such as sorption of water on the throats' surface (Bakhshian and Sahimi, 2017). We have not included such secondary effects in the simulation, because our primary interest is salt precipitation and the effect of the pore space heterogeneity on the phenomenon. Depending on the morphology porous medium and the chemical composition of its pores' surface, sorption of water might have some effect on the last stage of drying. On the other hand, through comprehensive experiments, Bergstad and Shokri (2016), who studied evaporation of brine in sand grains, reported drying curves and the images of the phase distributions that are comparable with what we are reporting in the present paper, even though they utilized porous media with various structures.

Another important characteristic of drying in porous media is the length $L_{D}$ and shape of its front. Figure 13 presents the time evolution of $L_{D}(t)$ in the six types of porous media of Figs. $3-8$. It is clear that the morphology of the porous medium, and in particular its correlation structure, have a significant effect on $L_{D}(t)$. For example, the average drying length is larger when the strata are parallel to the $y$ direction. Results shown in Fig. 13 indicates that at practically all times, $L_{D}(t)$ is larger for $\beta_{x} / \beta_{y}=1 / 4$; that is, when the strata are parallel to the $y$ direction. The physical effect of this type of morphology was already discussed. 
One may also study the effect of the pore space morphology on salt precipitation by considering the distribution of the throats' sizes that are partially or completely plugged by salt precipitation. Figure 14 depicts such distributions in the same six types of porous media that are shown in Figs. 3-8. Although the shapes of the distributions are more or less similar before and after the precipitation, there are also quantitative differences between the two. In Table 1 we also present the average throat sizes before and after salt precipitation. The simulations indicate that the porosity reduction due to precipitation is around $15 \%$, which is close to what have been reported for injection of $\mathrm{CO}_{2}$ in oil fields, $\approx 20$ percent. The results shown in Fig. 14 should also be compared with those shown in Fig. 15, which presents the PSDs before and after salt precipitations in a completely random (uncorrelated) porous medium.

Miri et al. (2015) studied salt precipitation during $\mathrm{CO}_{2}$ injection, using a fabricated $2 \mathrm{D}$ PN, initially saturated with brine. Similar to our PN the connectivity of their PN was 4, but their boundary conditions were different. They injected $\mathrm{CO}_{2}$ from one side and produced brine from the opposite side. The rate of injection was very low, with most of the water leaving the PN through evaporation. Despite the differences in the boundary conditions, Miri et al. (2015) reported uniform salt precipitation in their experiments, similar to what we report here. Experimental results reported by Kim et al. (2013) also share similarities with our results. They used fabricated 3D PNs to study drying and salt precipitation, and reported formation of uniform large salt crystals away from the vapor-liquid interface inside the porous medium.

\section{Summary}

This paper presented a pore-network model of isothermal evaporation of brine flowing in a porous medium and the resulting salt precipitation. Vapor diffusion in the gas phase, flow of brine, transport of salt and its precipitation in the pore space were all accounted for by the model. The dynamic evolution of the brine saturation, the evaporation rate, the length of the drying front, and the size distribution of the plugged or partially-plugged throats were computed and studied. Furthermore, we demonstrated the strong effect of the heterogeneity and correlations between the size of the throats on the evaporation rate and the various drying stages.

Despite extensive experimentation, the knowledge about factors that contribute to the phenomenon is still incomplete (Miri and Hellevang, 2016). The PN model provides a framework 
to study salt precipitation under more complex conditions than what we have considered in this paper. In particular, the lithology of geological formations possesses complex chemistry that affects the wettability, which in turn influences not only salt precipitation, but also the drying behavior (Or et al., 2013). Such effects will be included in the PN model, which we hope to report in the near future.

\section{Acknowledgments}

Work at USC was supported as part of the Center for Geologic Storage of $\mathrm{CO}_{2}$, an Energy Frontier Research Center funded by the U.S. Department of Energy, Office of Science, Basic Energy Sciences, under Award DE-SC0C12504. N.S. acknowledges funding by the the Leverhulme Trust (RPG-2014-331). 


\section{References}

Ananthakrishnan, V., Gill, W.N., Barduhn, A.J., 1965. Laminar dispersion in capillaries: Part I. Mathematical analysis. AIChE J. 11, 1063.

Ansari-Rad, M., Vaez Allaei, S.M., Sahimi, M., 2012. Nonuniversality of roughness exponent of quasistatic fracture surfaces. Phys. Rev. E 85, 021121.

Aris, R., 1956. On the dispersion of a solute in a fluid flowing through a tube. Proc. R. Soc. Lond. A 235, 67.

Bacci, G., Korre, A., Durucan, S., 2011. Experimental investigation into salt precipitation during $\mathrm{CO}_{2}$ injection in saline aquifers. Energy Procedia 4, 4450.

Bakhshian, S., Sahimi, M., 2017. Adsorption-induced swelling of porous media. Int. J. Greenh. Gas Control 57, 1-13.

Battistelli, A., Calore, C., Pruess, K., 1997. The simulator tough2/ewasg for modelling geothermal reservoirs with brines and non-condensible gas. Geothermics 26, 437.

Baumann, G., Henninges, J., De Lucia, M., 2014. Monitoring of saturation changes and salt precipitation during $\mathrm{CO}_{2}$ injection using pulsed neutron-gamma logging at the Ketzin pilot site. Int. J. Greenhouse Gas Control 28, 134.

Bergstad, M., Shokri, N., 2016. Evaporation of $\mathrm{NaCl}$ solution from porous media with mixed wettability. Geophys. Res. Lett. 43, DOI: 10.1002/2016GL068665.

Bird, R.B., Stewart, W.E., Lightfoot, E.N., 2007, Transport Phenomena, 2nd corrected ed., New York, Wiley, Chapter 18.

Blunt, M.J., 2017. Multiphase Flow in Permeable Media, Camridsge University Press, London.

Blunt, M.J., Bijeljic, B., Dong, H., Gharbi, O., Iglauer, S., Mostaghimi, P., Paluszny, A., Pentland, C., 2013. Pore-scale imaging and modelling. Adv. Water Resour. 51, 197.

Blunt, M.J., Jackson, J.D., Piri, M., Valvatne, P.H., 2002. Detailed physics, predictive capbilities and macroscopic consequences for pore-network models of multiphase flow. Adv. Water Resour. 25, 1069. 
Bönhorst, M., Walzel, P., Rahimi, A., Kharaghani, A., Tsotsas, E., Nestle, N., Besser, A., Kleine Jäger, F., Metzger, T., 2016. Influence of pore structure and impregnation drying conditions on the solid distribution in porous support materials. Drying Technol. 34, 1964.

Celia, M.A., Nordbotten, J.M., Court, B., Dobossy, M., Bachu, S., 2011. Field-scale application of a semi-analytical model for estimation of $\mathrm{CO}_{2}$ and brine leakage along old wells. Int. J. Greenhouse Gas Control 5, 257.

Chen, F., Mourhatch, R., Tsotsis, T.T., Sahimi, M., 2008. Pore network model of transport and separation of binary gas mixtures in nanoporous membranes. J. Membr. Sci. 315, 48.

Daian, J.F., Saliba, J., 1991. Determining a representative random pore-network for moisture sorption and migration in cement mortar. Int. J. Heat Mass Transfer 34, 2081.

Desarnaud, J., Derluyn, H., Carmeliet, J., Bonn, D., Shahidzadeh, N., 2014. Metastability limit for the nucleation of $\mathrm{NaCl}$ crystals in confinement. J. Phys. Chem. Lett. 5, 890.

Dashtian, H., Jafari, G.R., Sahimi, M., Masihi, M., 2011. Scaling, multifractality, and longrange correlations in well log data of large-scale porous media. Physica A 390, 2096.

Dashtian, H., Wang, H., Sahimi, M., 2017. Nucleation of salt crystals in clay minerals: molecular dynamics simulation. J. Phys. Chem. Lett. 8, 3166.

Dashtian, H., Yang, Y., Sahimi, M., 2015. Nonuniversality of the Archie exponent due to multifractality of resistivity well logs. Geophys. Res. Lett. 42, 10655.

Ebrahimi, F., 2010. Invasion percolation: A computational algorithm for complex phenomena. Comput. Sci. Eng. 12(2), 84.

Flatt, R.J., 2002. Salt damage in porous materials: how high supersaturations are generated. J. Crystal Growth 242, 435.

Ghassemzadeh, J., Hashemi, M., Sartor, L., Sahimi, M., 2001. Pore network simulation of fluid imbibition into paper during coating processes: I. Model development. AIChE J. $47,519$. 
Ghassemzadeh, J., Sahimi, M., 2004. Pore network simulation of fluid imbibition into paper during coating II: characterization of paper's morphology and computation of its effective permeability tensor. Chem. Eng. Sci. 59, 2265.

Guglielmini, L., Gontcharov, A., Aldykiewicz Jr., A.J., Stone, A.H., 2008. Drying of salt solutions in porous materials: intermediate-time dynamics and efflorescence. Phys. Fluids 20, 077101 .

Hekmatzadeh, M., Dadvar, M., Sahimi, M., 2016. Pore-network simulation of unstable miscible displacements in porous media. Transp. Porous Med. 113, 511.

Hewett, T.A., 1986. Fractal distributions of reservoir heterogeneity and their influence on fluid transport. SPE Paper 15386, New Orleans, Louisiana.

item Huinink, H.P., Pel, L., Michels, M.A.J., 2002, How ions distribute in a drying porous medium: A simple model. Phys. Fluids 14, 1389.

Imdakm, A.O., Sahimi, M., 1987. Transport of large particles in flow through porous media. Phys. Rev. A 36, 5304.

Imdakm, A.O., Sahimi, M., 1991. Computer simulation of particle transport processes in flow through porous media. Chem. Eng. Sci. 46, 1977.

Jambhekar, V.A., Helmig, R., Schröder, N., Shokri, N., 2015. Free-flow-porous-media coupling for evaporation-driven transport and precipitation of salt in soil. Transp. Porous Med. $110,251$.

Kim, M., Sell, A., Sinton, D., 2013. Aquifer-on-a-chip: understanding pore-scale salt precipitation dynamics during $\mathrm{CO}_{2}$ sequestration. Lab. Chip 13, 2508.

Knackstedt, M.A., Sahimi, M., Sheppard, A.P., 2000. Invasion percolation with long-range correlations: First-order phase transition and nonuniversal scaling properties. Phys. Rev. E 61, 4920.

Knackstedt, M.A., Sheppard, A.P., Pinczewski, W.V., 1998. Simulation of mercury porosimetry on correlated grids: evidence for extended correlated heterogeneity at the pore scale in rock. Phys. Rev. E 58, 6923. 
Knackstedt, M.A., Sheppard, A.P., Sahimi, M., 2001. Pore network modelling of two-phase flow in porous rock: the effect of correlated heterogeneity. Adv. Water Resour. 24, 257.

Koplik, J., Redner, S., Wilkinson, D., 1988. Transport and dispersion in random networks with percolation disorder. Phys. Rev. A 37, 2619.

Laurindo, J.B., Prat, M., 1998. Modelling of drying in capillary porous media: a discrete approach. Drying Technol. 16, 1769.

Le, D., Hoang, H., Mahadevan, J., 2009. Impact of capillary-driven liquid films on salt crystallization, Transp. Porous Med. 80, 229.

Le Bray, Y., Prat, M., 1999. Three-dimensional pore network simulation of drying in capillary porous media. Int. J. Heat Mass Transfer 42, 4207.

Lehmann, P., Assouline, S., Or, D., 2008. Characteristic lengths affecting evaporative drying of porous media. Phys. Rev. E 77, 056309.

Mahadevan, J., Sharma, M.M., Yortsos, Y.C., 2006. Flow-through drying of porous media. AIChE J. 52, 2367.

Malmir, H., Sahimi, M., Rahimi Tabar, M.R., 2016(a). Microstructural characterization of random packings of cubic particles. Sci. Rep. 6, paper 35024.

Malmir, H., Sahimi, M., Rahimi Tabar, M.R., 2016(b). Packing of nonoverlapping cubic particles: computational algorithms and microstructural characteristics. Phys. Rev. E 94, 062901.

Malmir, H., Sahimi, M., Rahimi Tabar, M.R., 2017. Statistical characterization of microstructure of packings of polydisperse hard cubes. Phys. Rev. E 95, 052902.

Marion, G.M., 2001. Carbonate mineral solubility at low temperatures in the NaK MgCaH$\mathrm{ClSO}_{4} \mathrm{OHHCO}_{3} \mathrm{CO}_{3} \mathrm{CO}_{2} \mathrm{H}_{2} \mathrm{O}$ system. Geochim. Cosmochim. Acta 65, 1883.

Mehmani, Y., Balhoff, M.T., 2015. Eulerian network modeling of longitudinal dispersion. Water Resour. Res. 51, 8586. 
Metzger, T., Tsotsas, E., Prat, M., 2007. Pore network models: a powerful tool to study drying at the pore level, in, Modern Drying Technology, Volume 1: Computational Tools at Different Scales, E. Tsotsas and A.S. Mujumdar, eds., Wiley, Weinheim, p. 57.

Miri, R., Hellevang, H., 2016. Salt precipitation during $\mathrm{CO}_{2}$ storage: a review. Int. J. Greenhouse Gas Control 51, 136.

Miri, R., van Noort, R., Aagaard, P., Hellevang, H., 2015. New insights on the physics of salt precipitation during injection of $\mathrm{CO} 2$ into saline aquifers. Int. J. Greenhouse Gas Control 43, 10 .

Mourhatch, R., Tsotsis, T.T., Sahimi, M., 2010a. Network model for the evolution of the pore structure of silicon-carbide membranes during their fabrication. J. Membr. Sci. 356, 138.

Mourhatch, R., Tsotsis, T.T., Sahimi, M., 2010b. Determination of the true pore size distribution by flow permporometry experiments: An invasion percolation algorithm. J. Membr. Sci. 367, 53 .

Mukhopadhyay, S., Sahimi, M., 2000. Calculation of the effective permeabilities of field-scale porous media. Chem. Eng. Sci. 55, 4495.

Muller, N., Qi, R., Mackie, E., Pruess, K., Blunt, M.J., 2009. $\mathrm{CO}_{2}$ injection impairment due to halite precipitation. Energy Procedia 1, 3507.

Nachshon, U., Weisbrod, N., Dragila, M.I., Grader, A., 2001. Combined evaporation and salt precipitation inhomogeneous and heterogeneous porous media. Water Resour. Res. 47, W03513.

Nachshon U., Shahraeeni, A., Or, D., Dragila, M., Weisbrod, N., 2011. Infrared thermography of evaporative fluxes and dynamics of salt deposition on heterogeneous porous surfaces. Water Resour. Res. 47, W12519.

Neuman, S.P. (1994). Generalized scaling of permeabilities: validation and effect of support scale. Geophys. Res. Lett. 21, 349.

Noiriel, C., Renard, F., Doan, M.L., Gratier, J.P., 2010. Intense fracturing and fracture sealing induced by mineral growth in porous rocks. Chem. Geol. 269, 197. 
Norouzi Rad, M., Shokri, N., 2012. Nonlinear effects of salt concentrations on evaporation from porous media. Geophys. Res. Lett. 39, L04403.

Norouzi Rad, M., Shokri, N., 2014. Effects of grain angularity on $\mathrm{NaCl}$ precipitation in porous media during evaporation. Water Resour. Res. 50, 9020.

Norouzi Rad, M., Shokri, N., Sahimi, M., 2013. Pore-scale dynamics of salt precipitation in drying porous media. Phys. Rev. E 88, 032404.

Or, D., Lehmann, P, Shahraeeni, E., Shokri, N., 2013. Advances in soil evaporation physics: A review. Vadose Zone J. 12(4), 1.

Ott, H., de Kloe, K., Marcelis, F., Makurat, A., 2011. Injection of supercritical $\mathrm{CO}_{2}$ in brine saturated sandstone: pattern formation during salt precipitation. Energy Procedia 4, 4425.

Peysson, Y., Andre, L., Azaroual, M., 2014. Well injectivity during $\mathrm{CO}_{2}$ storage operations in deep saline aquiferspart 1: experimental investigation of drying effects, salt precipitation and capillary forces. Int. J. Greenhouse Gas Control 22, 291.

Piri, M., Blunt, M.J., 2005a. Three-dimensional mixed-wet random pore-scale network modeling of two- and three-phase flow in porous media. I. Model description. Phys. Rev. E 71, 026301.

Piri, M., Blunt, M.J., 2005b. Three-dimensional mixed-wet random pore-scale network modeling of two- and three-phase flow in porous media. II. Results. Phys. Rev. E 71, 026302 .

Prat, M., 1995. Isothermal drying of non-hygroscopic capillary-porous materials as an invasion percolation process. Int. J. Multiphase Flow 21, 875.

Rajabbeigi, N., Tsotsis, T.T., Sahimi, M., 2009. Molecular pore-network model for nanoporous materials. II: Application to transport and separation of gaseous mixtures in siliconcarbide membranes. J. Membr. Sci. 345, 323. 
Roels, S.M., Ott, H., Zitha, P.L., 2014. $\mu$-CT analysis and numerical simulation of drying effects of $\mathrm{CO} 2$ injection into brine-saturated porous media. Int. J. Greenhouse Gas Control 27, 146.

Sahimi, M., 2011. Flow and Transport in Porous Media and Fractured Rock, 2nd ed. Wiley$\mathrm{VCH}$, Weinham.

Sahimi, M., Imdakm, A.O., 1991. Hydrodynamics of particulate motion in porous media. Phys. Rev. Lett. 66, 1169.

Sahimi, M., Mukhopadhyay, S., 1996. Scaling properties of a percolation model with longrange correlations. Phys. Rev. E 54, 3870.

Scherer, G.W., 1990. Theory of drying. J. Am. Ceram. Soc. 73, 3.

Schiro, M., Ruiz-Agudo, E., Rodriguez-Navarro, C., 2012. Damage mechanisms of porous materials due to in-pore salt crystallization. Phys. Rev. Lett. 109, 265503.

Schoups, G., Hopmans, J.W., Young, C.A., Vrugt, J.A., Wallender, W.W., Tanji, K.K., Panday, S., 2005. Sustainability of irrigated agriculture in the San Joaquin valley, California. Proc. Natl. Acad. Sci. USA 102, 15352.

Sghaier, N., Geoffroy, N., Prat, M., Eloukabi, H., Ben Nasrallah, S., 2014. Evaporation-driven growth of large crystallized salt structures in a porous medium. Phys. Rev. E 90, 042402.

Shahidzadeh-Bonn, N., Desarnaud, J., Bertrand, F., Chateau, X., Bonn, D., 2010. Damage in porous media due to salt crystallization. Phys. Rev. E 81, 066110.

Shahraeeni, E., Or, D., 2010. Thermo-evaporative fluxes from heterogeneous porous surfaces resolved by infrared thermography. Water Resour. Res. 46, W09511.

Shahraeeni, E., Lehmann, P., Or, D., 2012. Coupling of evaporative fluxes from drying porous surfaces with air boundary layer: Characteristics of evaporation from discrete pores. Water Resour. Res. 48, W09525.

Shaw, M.T., 1987. Drying as an immiscible displacement process with fluid counterflow. Phys. Rev. Lett. 59, 1671. 
Shokri, N., 2014. Pore-scale dynamics of salt transport and distribution in drying porous media. Phys. Fluids 26, 012106.

Shokri, N., Lehmann, P., Or, D., 2010. Liquid-phase continuity and solute concentration dynamics during evaporation from porous media: Pore-scale processes near vaporization surface. Phys. Rev. E 81, 046308.

Shokri, N., Lehmann, P., Vontobel, P., Or, D., 2008. Drying front and water content dynamics during evaporation from sand delineated by neutron radiography. Water Resour. Res. 44, W06418.

Shokri, N., Or, D., 2011. What determines drying rates at the onset of diffusion controlled stage-2 evaporation from porous media? Water Resour. Res. 47, W09513.

Shokri, N., Sahimi, M., Or, D., 2012. Morphology, propagation dynamics and scaling characteristics of drying fronts in porous media. Geophys. Res. Lett. 39, L09401.

Shokri-Kuehni, S.M.S., Vetter, T., Webb, C., Shokri, N., 2017. New insights into saline water evaporation from porous media: Complex interaction between evaporation rates, precipitation and surface temperature. Geophys. Res. Lett. 44, 5504.

Sorbie, K.S., Clifford, P.J., 1991. The inclusion of molecular diffusion effects in the network modelling of hydrodynamic dispersion in porous media. Chem. Eng. Sci. 46, 2525.

Surasani, V.K., Metzger, T., Tsotsas, E., 2007. Consideration of heat transfer in pore network modelling of convective drying. Int. J. Heat Mass Transfer 51, 2506.

Taylor, G.I., 1953. Dispersion of soluble matter in solvent flowing slowly through a tube. Proc. Roy. Soc. Lond. A 219, 186.

Toner, J.D., Catling, D.C., Light, B., 2015. Modeling salt precipitation from brines on Mars: Evaporation versus freezing origin for soil salts. Icarus 250, 451.

Tsotsas, E., Mujumdar, A.S., edits., 2007. Modern Drying Technology, Volume 1: Computational Tools at Different Scales. Wiley, Weinheim. 
Veran-Tissoires, S., Prat, M., 2012. Discrete salt crystallization at the surface of a porous medium. Phys. Rev. Lett. 108, 054502.

Veran-Tissoires, S., Prat, M., 2014. Evaporation of a sodium chloride solution from a saturated porous medium with efflorescence formation. J. Fluid Mech. 749, 701.

Yiotis, A.G., Stubos, A.K., Boudouvis, A.G., and Y.C. Yortsos, Y.C., 2001. A 2-D Pore network model of drying of single-component liquids in porous media. Adv. Water Resour. 24, 439.

Yiotis, A.G., Tsimpanogiannis, I.N., Stubos, A.K., Yortsos, Y.C., 2006. Pore-network study of the characteristic periods in the drying of porous materials. J. Colloid Interface Sci. 297,738 .

Zeidouni, M., Pooladi-Darvish, M., Keith, D., 2008. Analytical solution to evaluate salt precipitation during $\mathrm{CO}_{2}$ injection in saline aquifers. Energy Procedia 1, 1775 . 
(a)

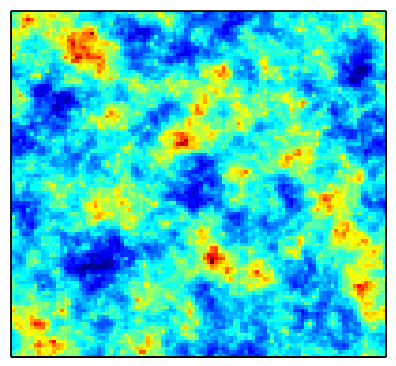

(d)

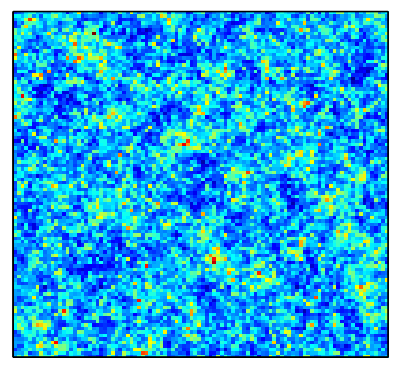

(b)

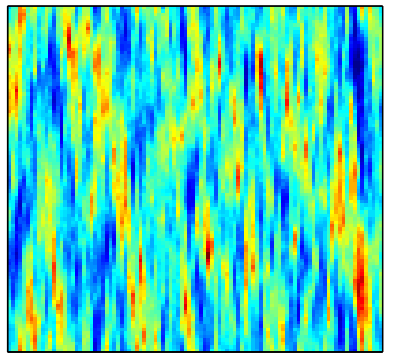

(e)

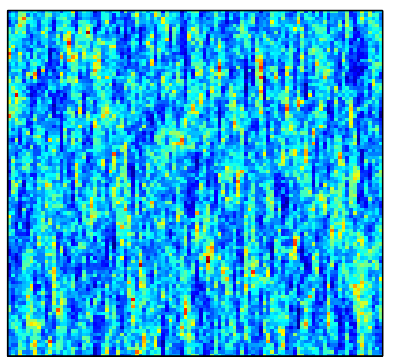

(c)

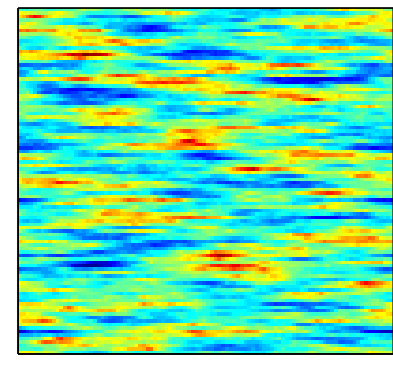

200
150

(f)

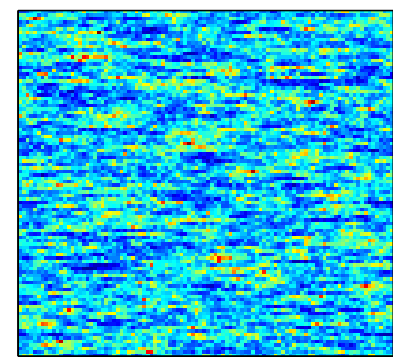

$-100$

50

Figure 1: Examples of pore networks with correlated throat sizes. The top and bottom rows show, respectively, the PNs with the Hurst exponents $H=0.7$ and 0.3 , and anisotropy $\beta_{x} / \beta_{y}=$ (a) and (d) 1; (b) and (e) 1/4, and (c) and (f) 4. 
1613

1614

1615

1616

1617

1618

1619

1620

1621

1622

1623

1624

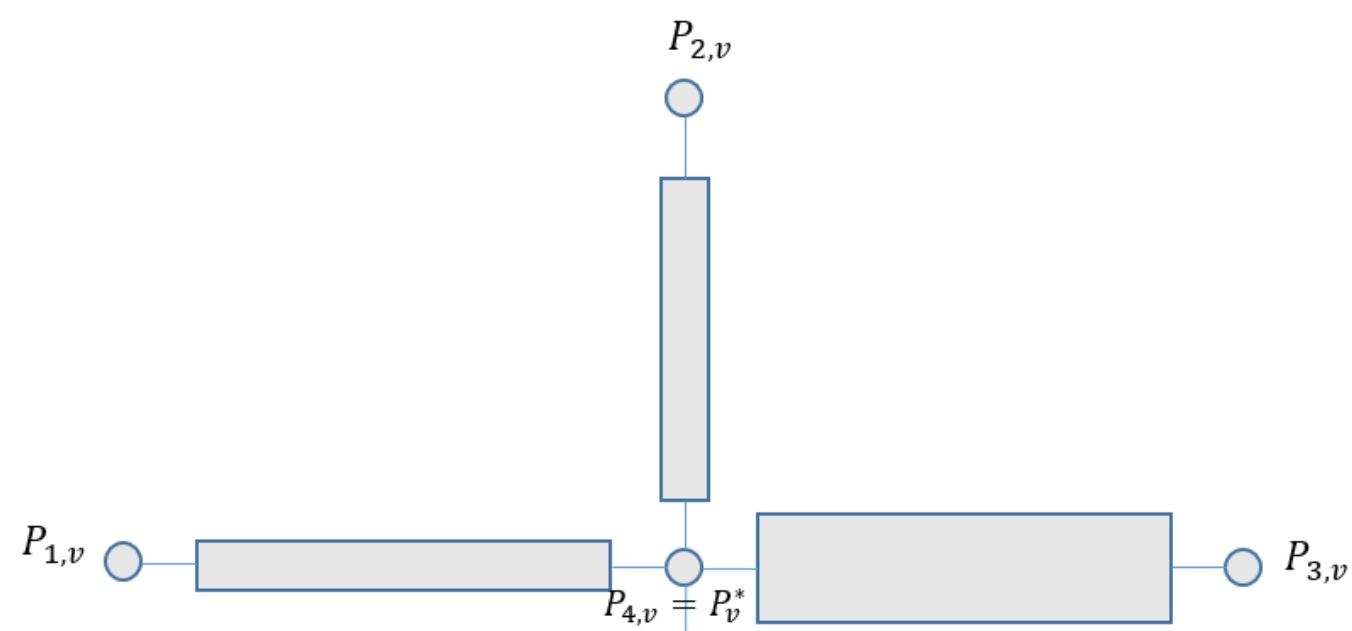

Figure 2: Illustration of the assumption of equilibrium vapor pressure in the central vapor pore, together with a neighboring throat containing liquid. 
(a)

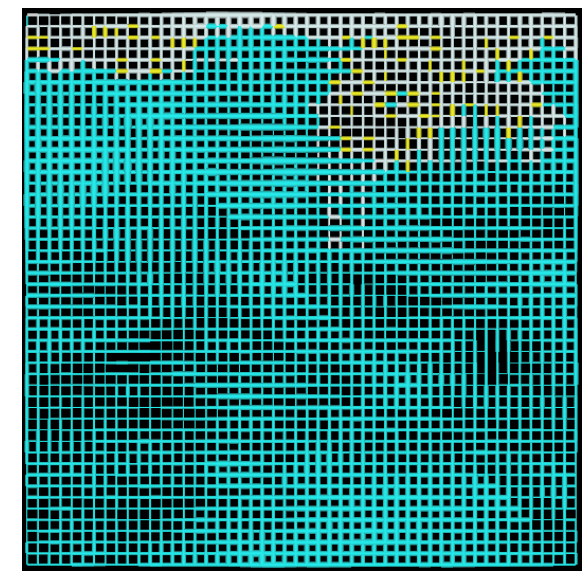

(c)

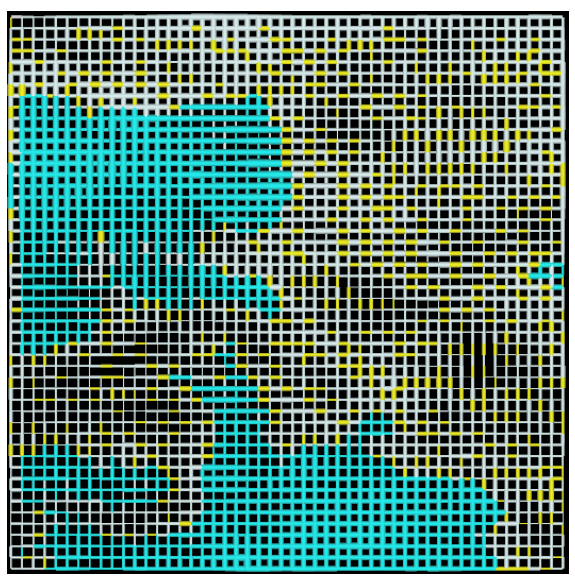

(b)

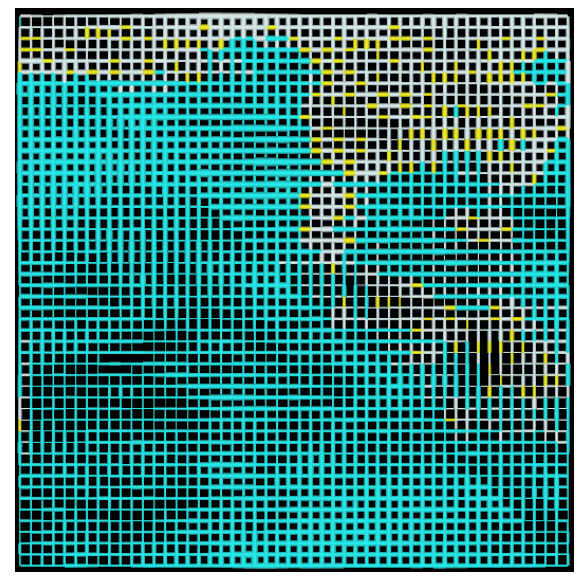

(d)

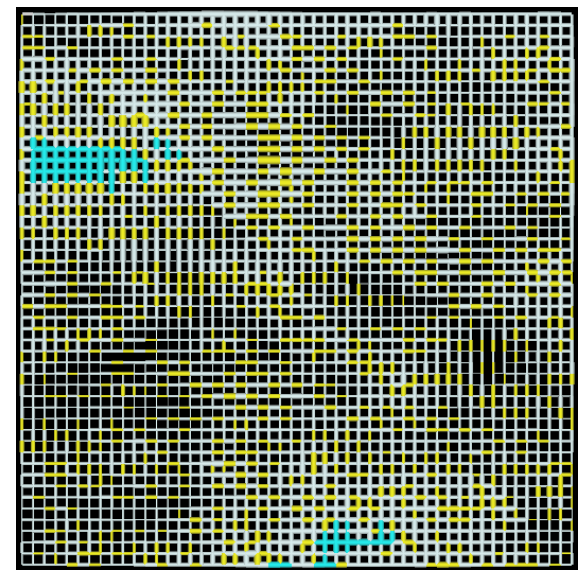

Figure 3: The phase distributions in an isotropic pore network with the Hurst exponent $H=0.7$ at time steps when vapor saturation is (a) 0.2 ; (b) 0.4 ; (c) 0.8 , and (d) 0.95 . Blue, white, and yellow indicate, respectively, the throats filled with brine, the emptied throats, and those that are partially or completely blocked. The top row of the network is open. 
(a)

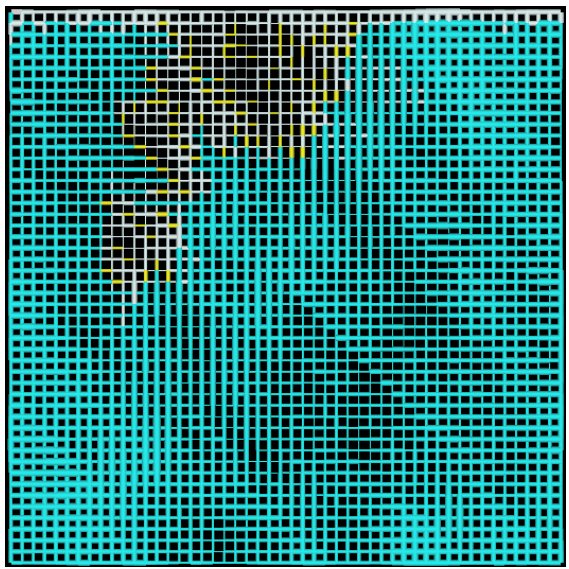

(c)

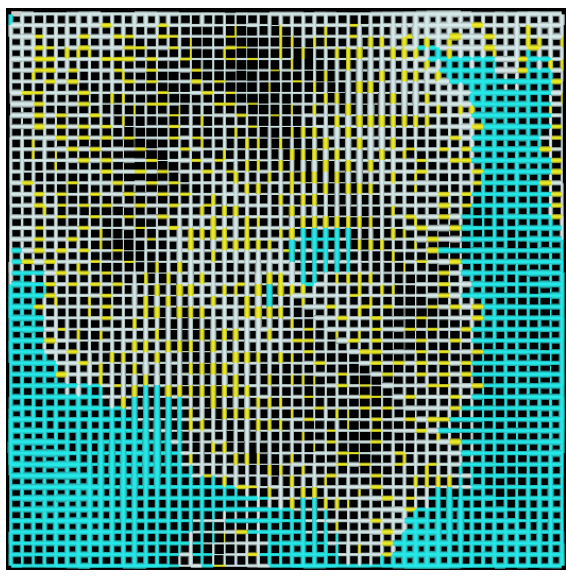

(b)

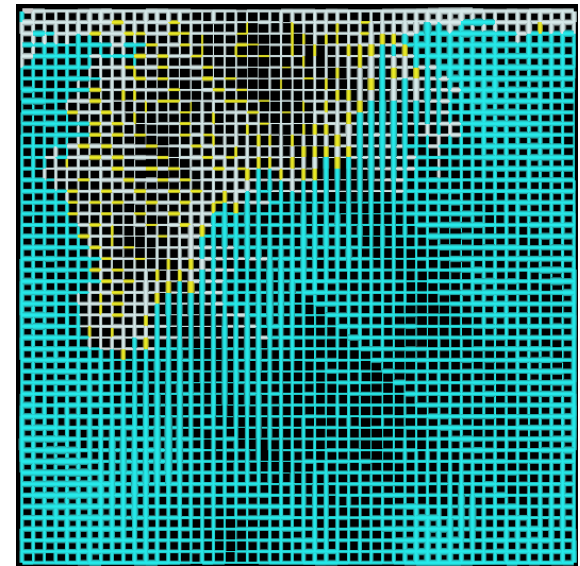

(d)

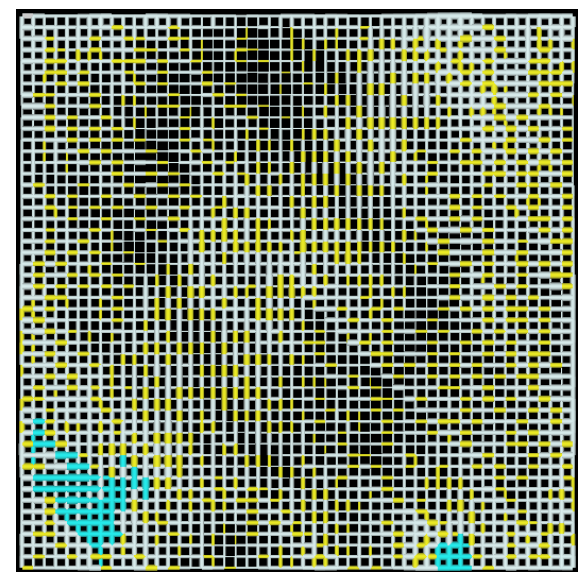

Figure 4: The phase distributions in a pore network with the Hurst exponent $H=0.7$ and anisotropy $\beta_{x} / \beta_{y}=1 / 4$, at time steps when vapor saturation is (a) 0.2 ; (b) 0.4 ; (c) 0.8 , and (d) 0.95. Colors indicate the same as in Fig. 3. The top row of the network is open. 
(a)

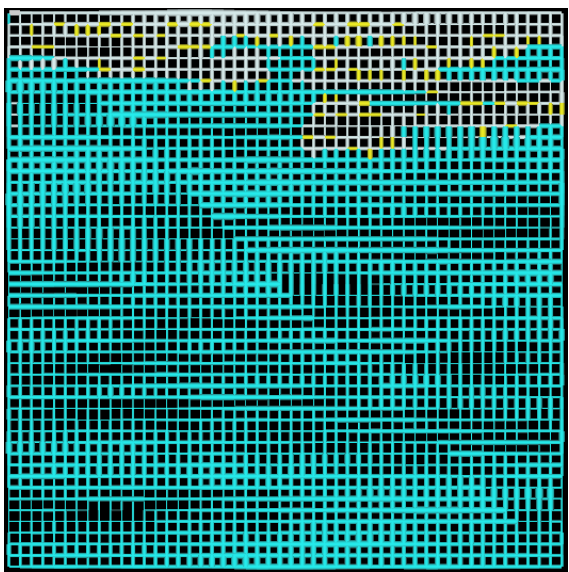

(c)

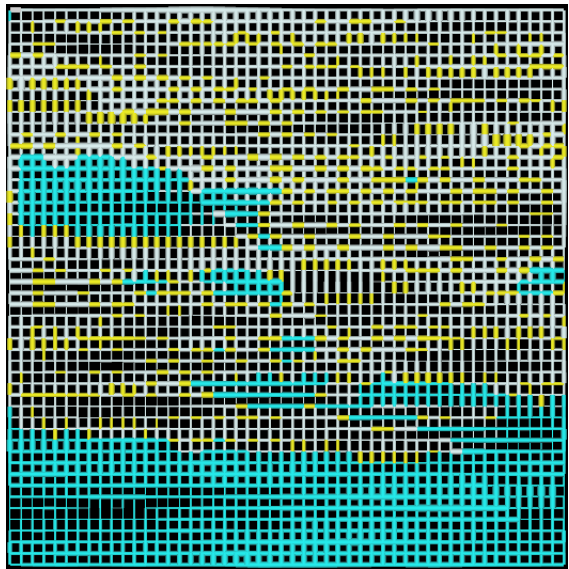

(b)

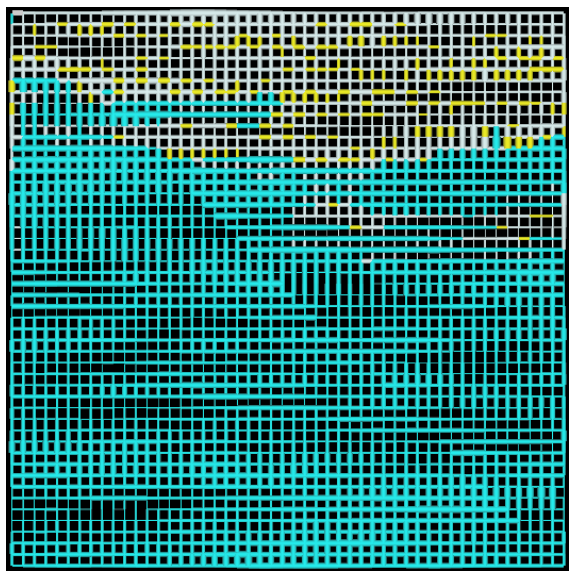

(d)

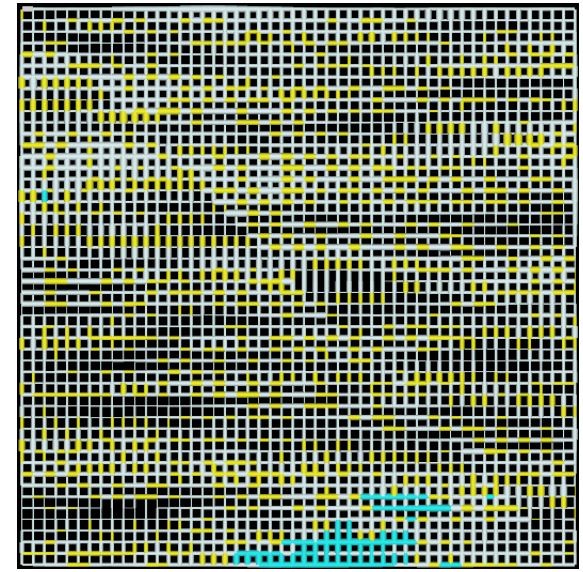

Figure 5: The phase distributions in a pore network with the Hurst exponent $H=0.7$ and anistropy $\beta_{x} / \beta_{y}=4$, at time steps when vapor saturation is (a) 0.2 ; (b) 0.4 ; (c) 0.8 , and (d) 0.95. Colors indicate the same as in Fig. 3. The top row of the network is open. 
(a)

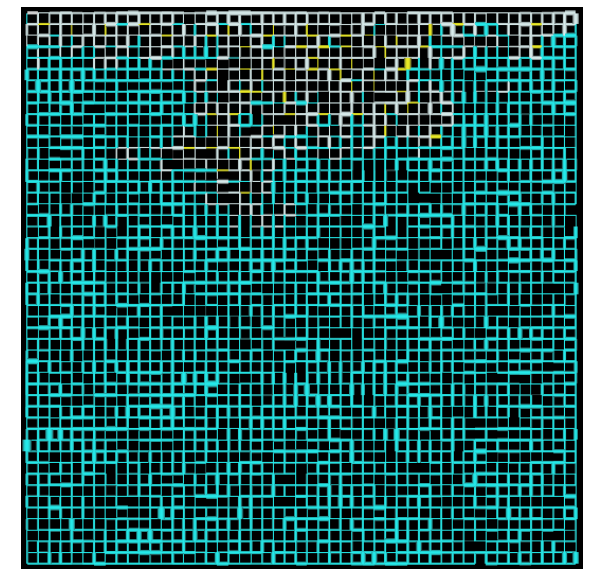

(c)

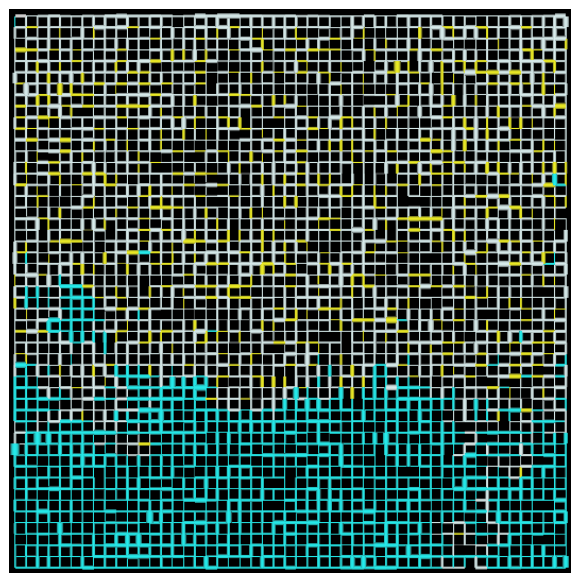

(b)

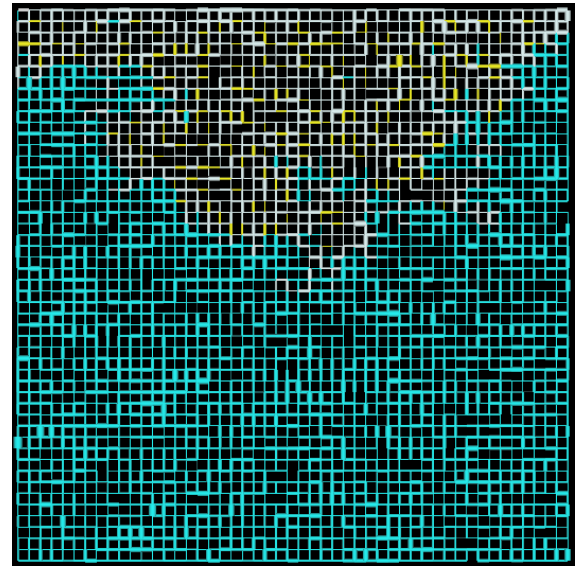

(d)

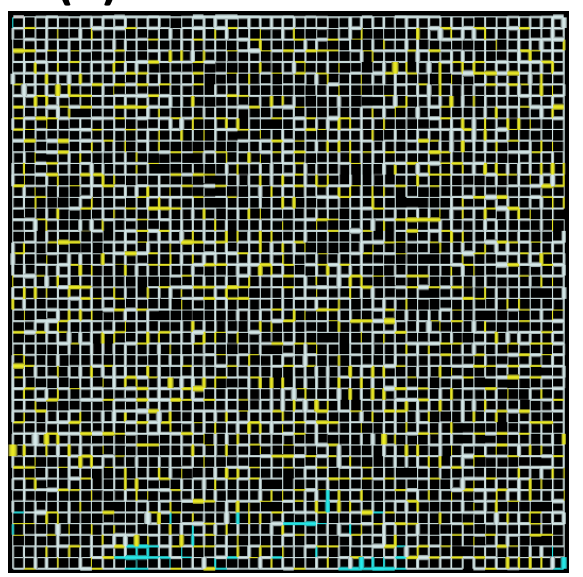

Figure 6: The phase distributions in an isotropic pore network with the Hurst exponent $H=0.3$ $\left(\beta_{x} / \beta_{y}=1\right)$, at time steps when vapor saturation is (a) 0.2 ; (b) 0.4 ; (c) 0.8 , and (d) 0.95. Colors indicate the same as in Fig. 3. The top row of the network is open. 
(a)

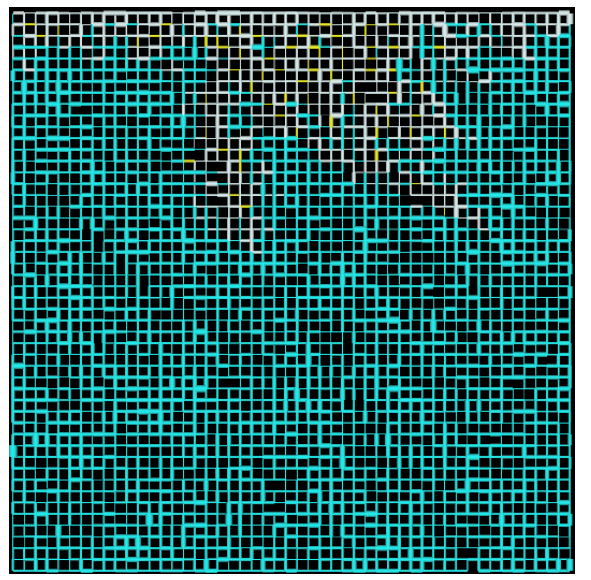

(c)

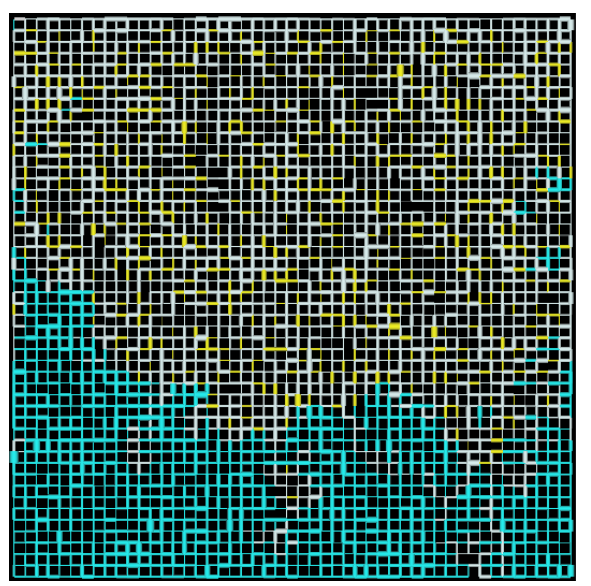

(b)

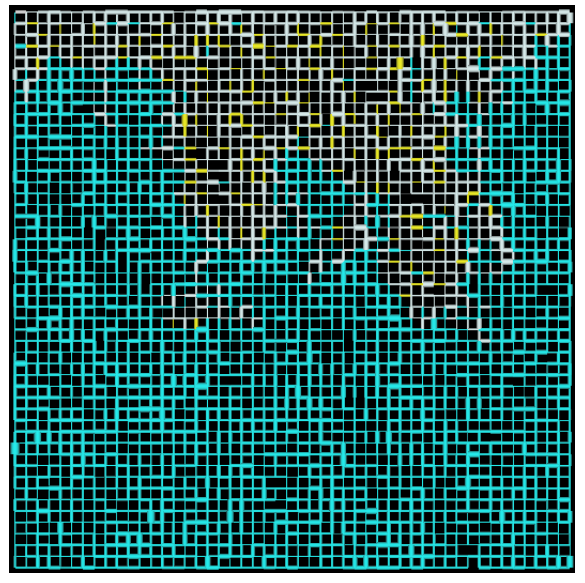

(d)

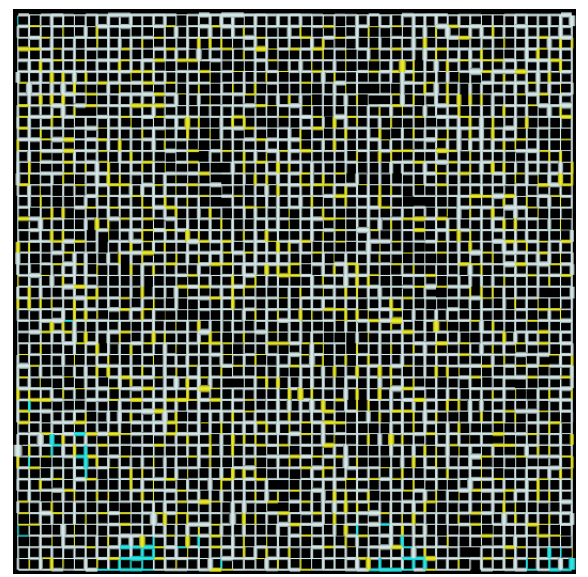

Figure 7: The phase distributions in a pore network with the Hurst exponent $H=0.3$ and anisotropy $\beta_{x} / \beta_{y}=1 / 4$, at time steps when vapor saturation is (a) 0.2 ; (b) 0.4 ; (c) 0.8 , and (d) 0.95. Colors indicate the same as in Fig. 3. The top row of the network is open. 
(a)

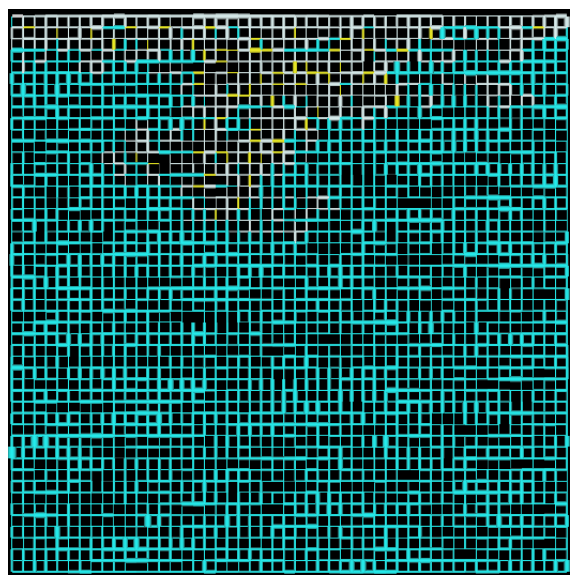

(c)

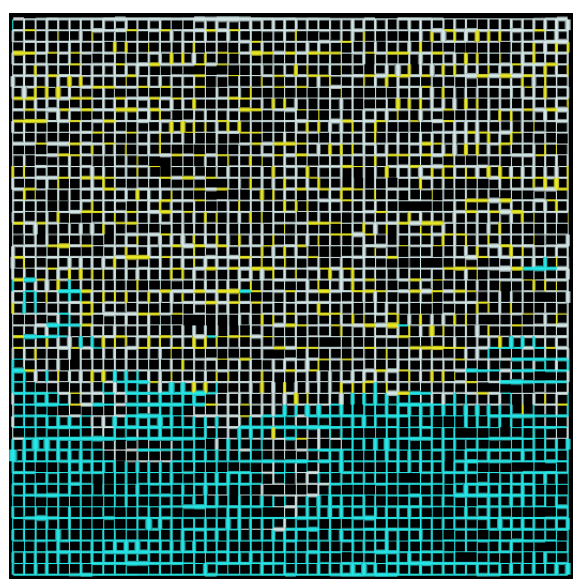

(b)

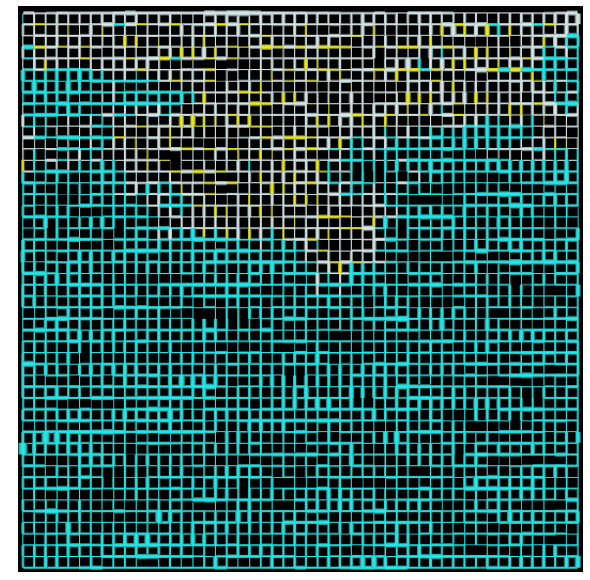

(d)

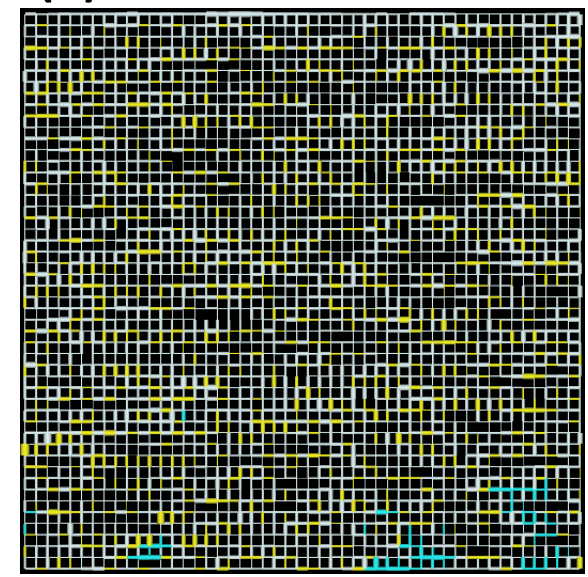

Figure 8: The phase distributions in a pore network with the Hurst exponent $H=0.3$ and anisotropy $\beta_{x} / \beta_{y}=4$, at time steps when vapor saturation is (a) 0.2 ; (b) 0.4 ; (c) 0.8 , and (d) 0.95. Colors indicate the same as in Fig. 3. The top row of the network is open. 
(a)

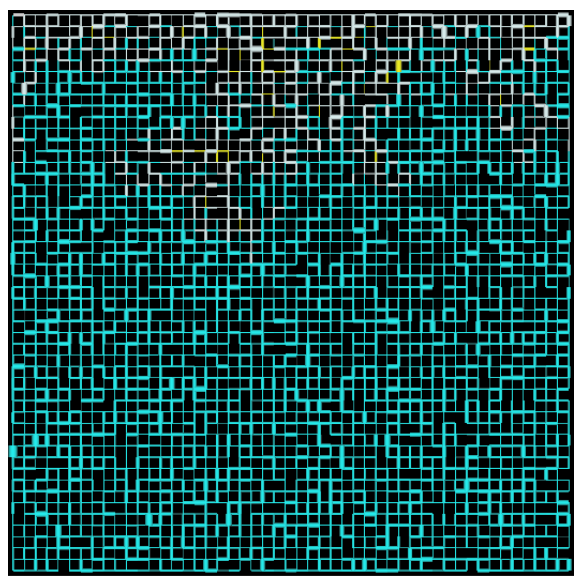

(c)

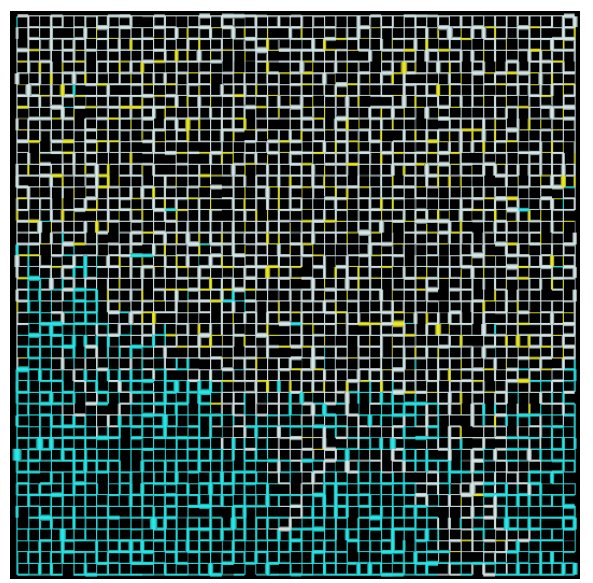

(b)

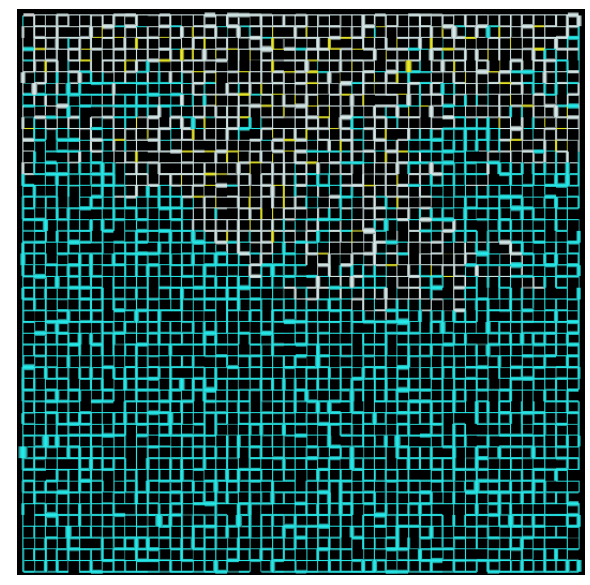

(d)

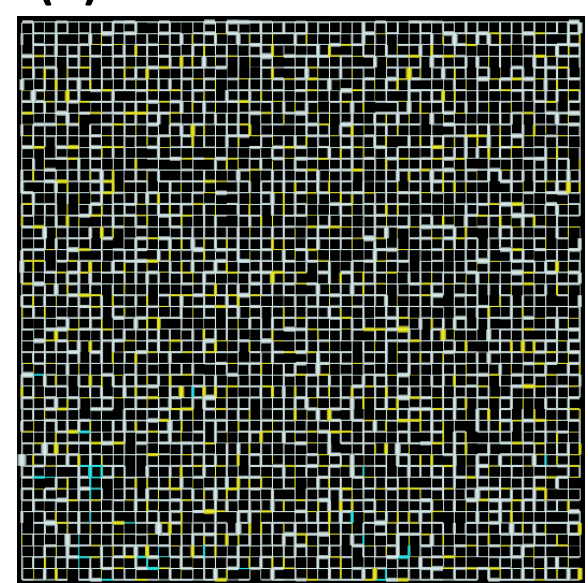

Figure 9: The phase distributions in an uncorrelated PN at time steps when vapor saturation is (a) 0.2 ; (b) 0.4 ; (c) 0.8 , and (d) 0.95. Colors indicate the same as in Fig. 3. The top row of the network is open. 

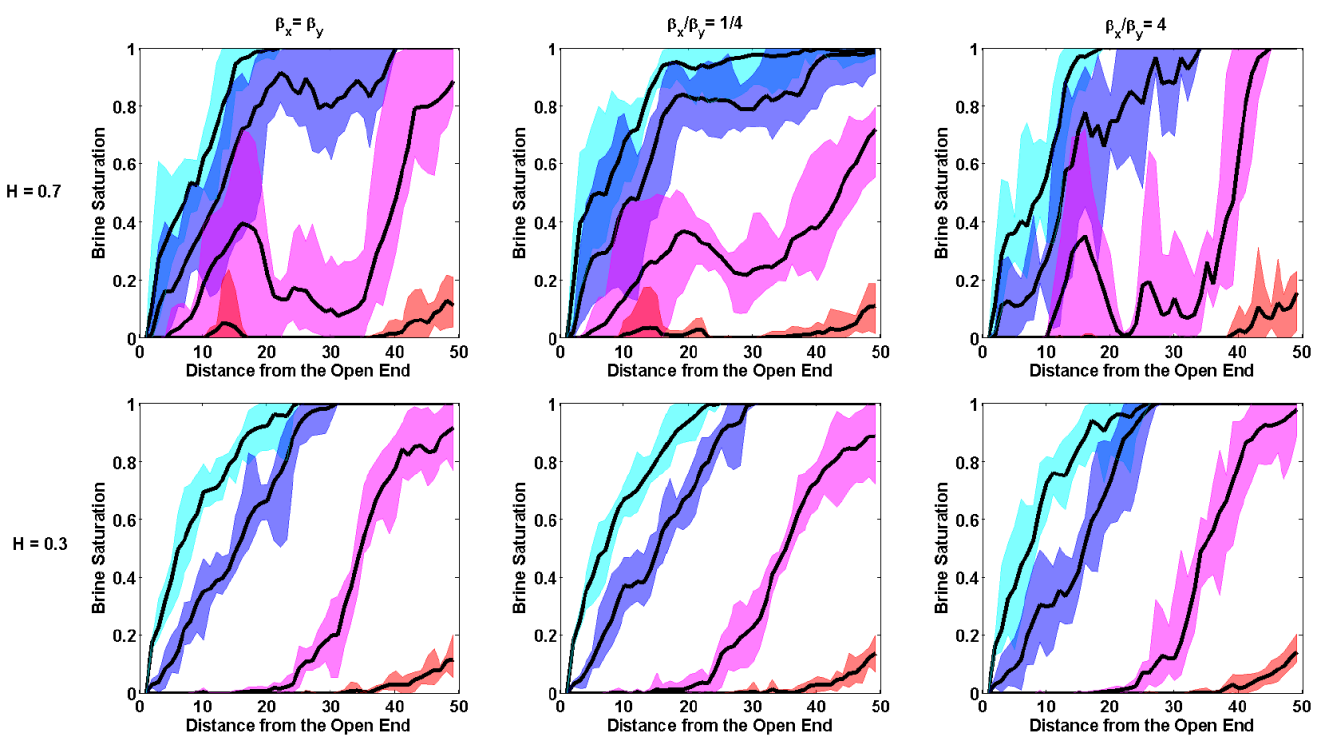

Figure 10: Dynamic evolution of the transversely-averaged brine saturations in six types of pore networks. Black curves show the average saturations; cyan, blue and pink shadings represent, respectively, the times at which vapor saturation is $0.2,0.4$, and 0.8 , while red indicates the time at which vapor saturation is 0.95 . The colored areas indicate the variations over multiple realizations. 


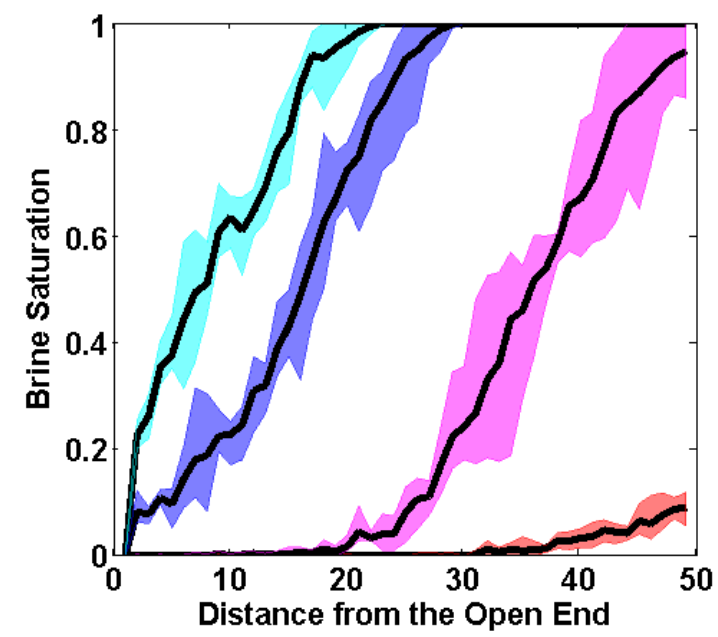

Figure 11: Dynamic evolution of the transversely-averaged saturations in the uncorrelated pore network. Black curves show the average saturations; cyan, blue and pink shadings represent, respectively, the times at which vapor saturation is $0.2,0.4$, and 0.8 , while red indicates the time at which vapor saturation is 0.95 . The colored areas indicated the variations over multiple realizations. 

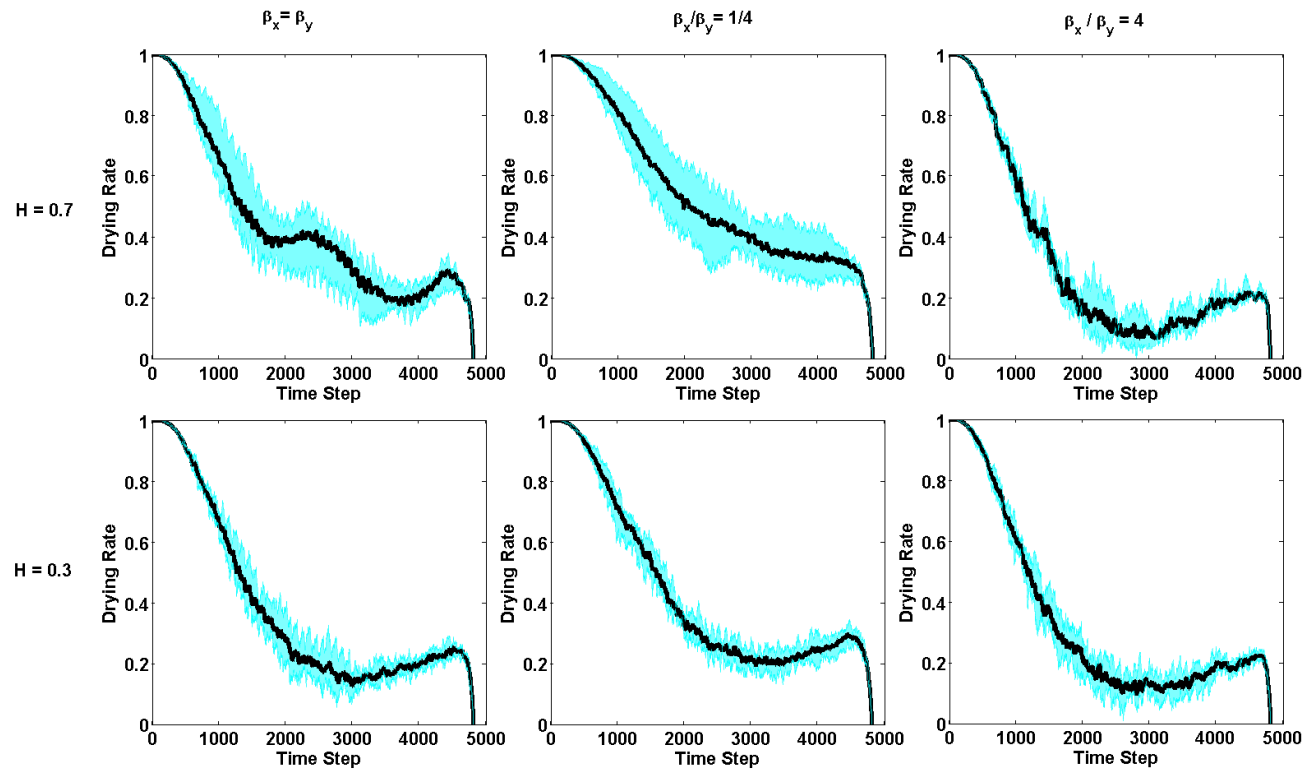

Figure 12: Dynamic evolution of the average drying rates (black curves), averaged over 10 realizations (shadings). The colored areas indicate variations over multiple realizations. 

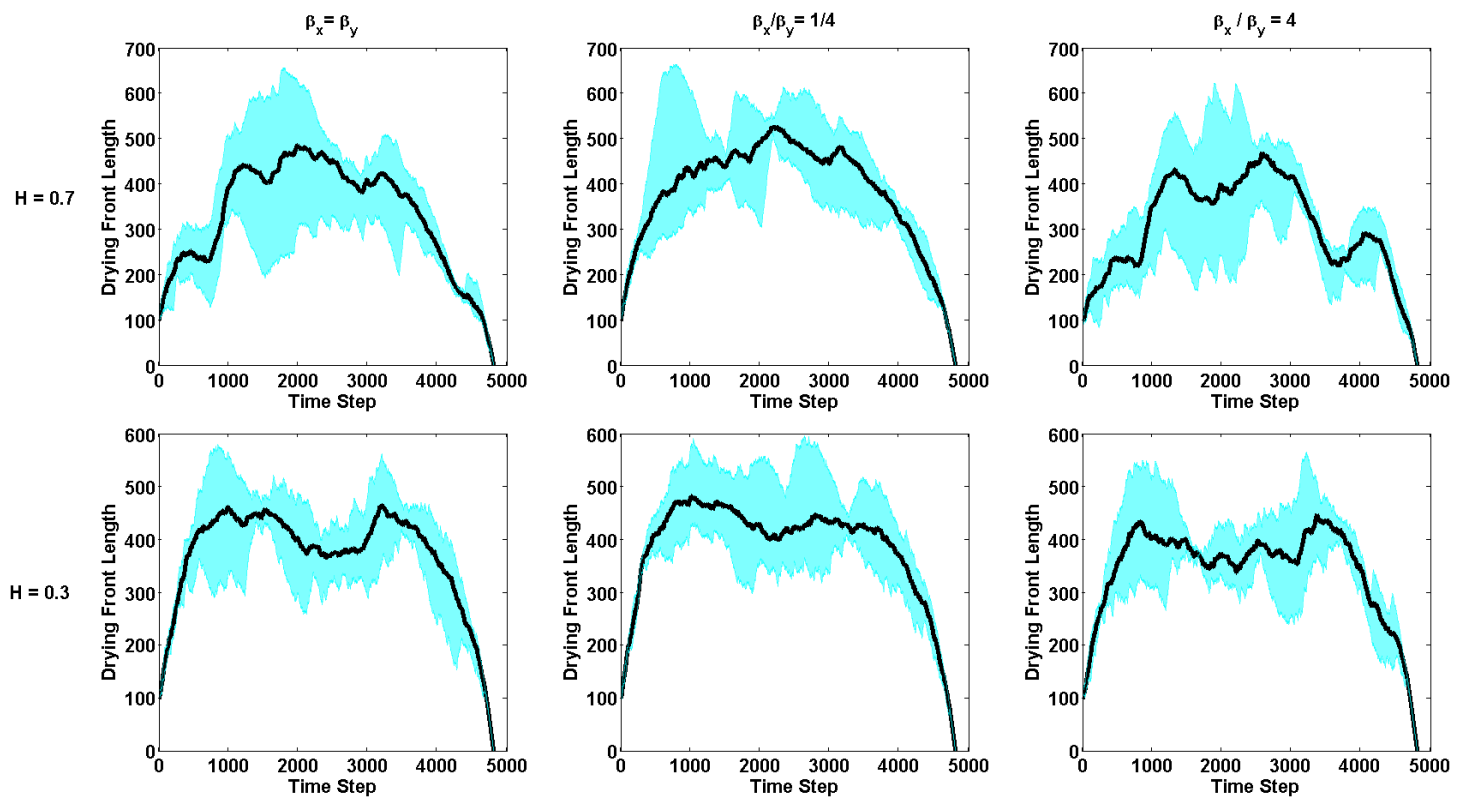

Figure 13: Dynamic evolution of the average length of the drying front. Shaded areas show the extent of the variations over ten realizations. 

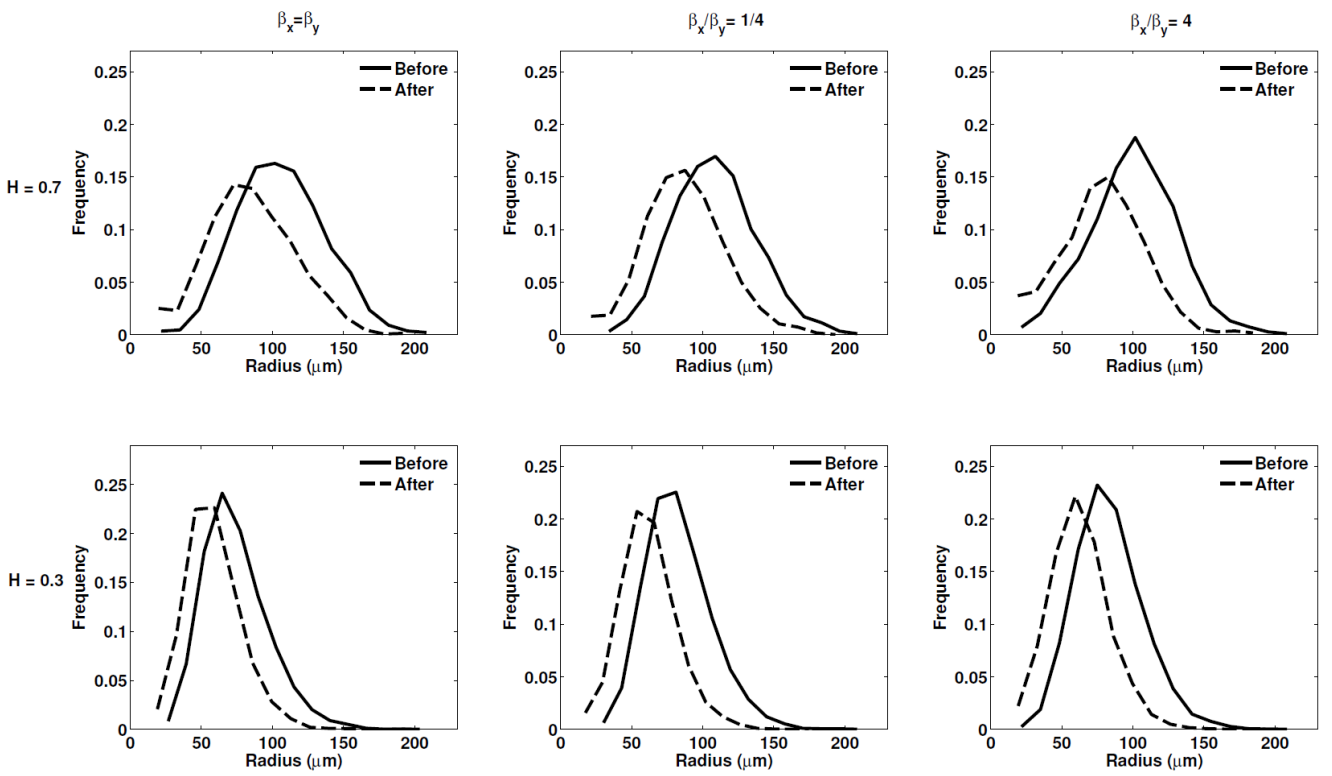

Figure 14: Size distribution of the partially or completely plugged throats before and after evaporation. 


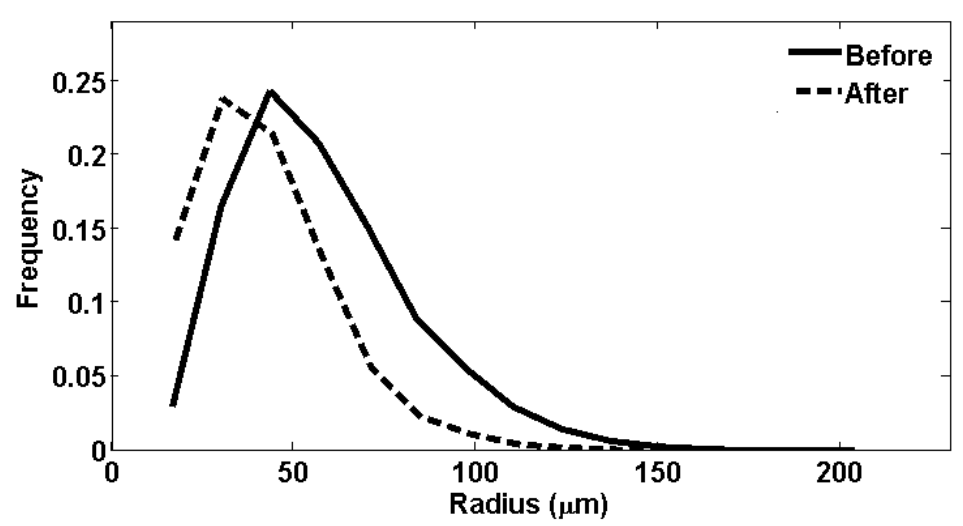

Figure 15: Size distribution of the partially or completely plugged throats before and after evaporation in the uncorrelated pore network. 